\title{
Discontinuous Galerkin Finite Element Convergence for Incompressible Miscible Displacement Problems of Low Regularity
}

\author{
Sören Bartels*, Max Jensen† Rüdiger Müller
}

March 4, 2008

\begin{abstract}
In this article we analyse the numerical approximation of incompressible miscible displacement problems with a combined mixed finite element and discontinuous Galerkin method under minimal regularity assumptions. The main result is that sequences of discrete solutions weakly accumulate at weak solutions of the continuous problem. In order to deal with the non-conformity of the method and to avoid overpenalisation of jumps across interelement boundaries, the careful construction of a reflexive subspace of the space of bounded variation, which compactly embeds into $L^{2}(\Omega)$, and of a lifting operator, which is compatible with the nonlinear diffusion coefficient, are required. An equivalent skew-symmetric formulation of the convection and reaction terms of the nonlinear partial differential equation allows to avoid flux limitation and nonetheless leads to an unconditionally stable and convergent numerical method. Numerical experiments underline the robustness of the proposed algorithm.
\end{abstract}

\section{Introduction}

Mathematical models which describe the miscible displacement of fluids in a porous medium have attracted considerable attention within the last two decades, motivated by the growing importance of the underlying physical processes in a variety of applications. Of particular economical relevance is the displacement of oil in underground reservoirs by fluids which mix with oil. Such enhanced displacement strategies improve the recovery rate significantly compared to classical oil recovery by water injection where capillary forces lead to a reduced sweep efficiency.

In this paper we study a system of nonlinear partial differential equations which models the single phase, miscible displacement of one fluid by another in a porous medium. With the assumption of incompressibility the system consists of an elliptic equation determining the pressure $p$ and Darcy velocity $u$ as well as of a parabolic equation which describes the evolution of the concentration $c$ of one fluid in the mixture. These two equations are coupled nonlinearly through the diffusion-dispersion coefficient, which depends on the Darcy velocity, through the viscosity of the fluid, which depends on the concentration, and through an advection term, which depends on the Darcy velocity. More precisely, the strong formulation of the model problem on the domain $\Omega_{T}:=(0, T) \times \Omega$ is

$$
\begin{aligned}
\phi \partial_{t} c-\operatorname{div}(\mathbb{D}(u) \nabla c)+u \cdot \nabla c+q^{I} c & =\hat{c} q^{I}, \\
\operatorname{div} u & =q^{I}-q^{P}, \\
u & =-\frac{\mathbb{K}}{\mu(c)}(\nabla p-\rho(c) g),
\end{aligned}
$$

\footnotetext{
${ }^{*}$ Institut für Numerische Simulation, Universität Bonn, Germany

${ }^{\dagger}$ Department of Mathematical Sciences, University of Durham, England

${ }^{\ddagger}$ Institut für Mathematik, Humboldt-Universität zu Berlin, Germany
} 
subject to the boundary conditions

$$
u \cdot n=0,(\mathbb{D}(u) \nabla c) \cdot n=0 \quad \text { on }(0, T) \times \partial \Omega,
$$

and the initial condition

$$
c(0, \cdot)=c_{0} .
$$

Here, $n$ is the outer unit normal vector on $\partial \Omega$ and $T$ is the time horizon. The functions $\phi$ and $\mathbb{K}$ model the porosity and the absolute permeability of the porous medium, respectively, $\mu$ and $\rho$ are the mobility (or viscosity) and the density of the fluid mixture, respectively, $g$ is a constant vector modelling gravity, $\mathbb{D}$ is the diffusion-dispersion coefficient, $\hat{c}$ and $c_{0}$ are the injected and the initial concentration, respectively. Finally, $q^{I}$ and $q^{P}$ are non-negative functions which represent injection well sources and production sinks, respectively.

We refer the reader to [F94, CE99] and references therein for detailed discussions concerning existence, uniqueness, and validity of a maximum principle for weak solutions of (1) to (5). While existence can be established under slightly more general assumptions [CE99] than the ones we employ and specify in (A1) to (A8) below, uniqueness of solutions is only known if $u$ admits additional regularity, e.g., if $u \in L^{\infty}\left(\Omega_{T}\right)$, see [F94]. A discussion of various generalisations of our mathematical model can be found in [F02].

The major goal of this paper is to contribute to closing the gap between analytical and numerical results for the model problem. Popular methods for solving (1) to (5) numerically employ non-conforming discrete spaces containing discontinuous functions and introduce additional terms to control jumps of numerical approximations, known as discontinuous Galerkin methods, see [SRW02, RW02]. These approaches are motivated by the convection dominated character of the concentration equation (1). While error estimates are available in the case of strong solutions [SRW02], whose existence is largely open, weak accumulation of approximations at weak solutions under minimum regularity assumptions has not been investigated yet. Since the construction of solutions in [F94, CE99] employs conforming spaces as well as approximation by regularised boundary value problems, these techniques cannot be employed to identify limits of numerical approximations obtained with the schemes mentioned above. We refer the reader to [AP98, EW80, DEW83] for other related numerical schemes to solve (1) to (5).

Key elements of our analysis are:

Discontinuous coefficients and corners: All coefficients of the partial differential equation except $\mu$ and $\rho$ may have discontinuities. Moreover, the domain $\Omega$ may have re-entrant corners. In the vicinity of corners and discontinuities the Darcy velocity typically exhibits singularities and is unbounded. Generally, it is not possible to predict the characteristics of these singularities precisely as $u$ is an unknown quantity and as $\mathbb{D}(u)$ grows linearly with $u$.

No 'cut-off' functionals: While 'cut-off' functionals have been used successfully to verify the existence of weak solutions [F94, CE99] they introduce a new, undefined parameter in numerical approximation schemes [SRW02]. We propose an alternative, skew-symmetric scheme for the advection term which circumvents these difficulties. A consequence of the skew-symmetric formulation is that the time discretisation is unconditionally stable.

Aubin-Lions compactness on a non-conforming space: The treatment of the nonlinearity in the convergence argument is based on an application of the Aubin-Lions lemma. We establish this compactness result on a non-conforming space $S$ instead of projecting the numerical solution onto the conforming space, thereby minimising the conditions on the mesh regularity.

Accumulation points of bounded $d G$ sequences: We demonstrate a number of regularity properties of accumulation points of $\mathrm{dG}$ sequences. In particular, energy-norm bounded $L^{2}$-limits of $\mathrm{dG}$ sequences have a gradient in $H^{1}(\Omega)$. The gradient can always be controlled without super-penalisation or similar techniques.

The article is organised as follows. In Section 2 we introduce the regularity assumptions and the weak formulation of the initial value problem. In Section 3 we fix notation and approximation spaces. In Section 4 we 
introduce the finite element method. In Section 5 we establish existence of numerical solutions and stability. In Section 6 we focus on general compactness properties of dG approximation spaces. This section can be read independently of Sections 4 and 5. In Section 7 we carry out the convergence analysis and verify that the limit of a subsequence is a weak solution. Finally, in Section 8 we investigate the behaviour of the scheme within the relevant parameter range by means of numerical experiments.

\section{The Initial Value Problem}

The following assumptions on the initial value problem are essential to our analysis and the statement of the weak formulation:

(A1) $\Omega \subset \mathbb{R}^{d}, d \in\{2,3\}$, is a bounded Lipschitz domain.

(A2) $\mathbb{K} \in L^{\infty}\left(\Omega ; \mathbb{R}^{d \times d}\right)$ and there exist positive real numbers $k_{\circ}, k^{\circ}$ such that

$$
k_{\circ}|\xi|^{2} \leq \xi^{\top} \mathbb{K}(x) \xi \leq k^{\circ}|\xi|^{2}
$$

for all $x \in \Omega$ and $\xi \in \mathbb{R}^{d}$. Moreover, $\mathbb{K}(x)$ is symmetric.

(A3) There exist positive real numbers $\mu_{\circ}, \mu^{\circ}$ such that the Lipschitz continuous function $\mu: \mathbb{R} \rightarrow \mathbb{R}$ satisfies

$$
\mu_{\circ} \leq \mu(c) \leq \mu^{\circ}
$$

for all $c \in \mathbb{R}$.

(A4) There exist positive real numbers $d_{\circ} \leq 1 \leq d^{\circ}$ such that the function

$$
\mathrm{D}: \mathbb{R}^{d} \times \Omega \rightarrow \mathbb{R}^{d \times d}
$$

satisfies the Carathéodory condition

$$
\begin{array}{ll}
\mathrm{D}(u, \cdot): x \mapsto \mathbb{D}(u, x) & \text { is measurable on } \Omega \text { for all } u \in \mathbb{R}^{d}, \\
\mathbb{D}(\cdot, x): u \mapsto \mathbb{D}(u, x) & \text { is continuous on } \mathbb{R}^{d} \text { for almost all } x \in \Omega,
\end{array}
$$

and the two-sided, $u$-dependent growth condition

$$
d_{\circ}(1+|u|)|\xi|^{2} \leq \xi^{\top} \mathbb{D}(u, x) \xi \leq d^{\circ}(1+|u|)|\xi|^{2}
$$

for all $u, \xi \in \mathbb{R}^{d}$ and $x \in \Omega$. Furthermore, $\mathbb{D}(u, x)$ is symmetric for $(u, x) \in \mathbb{R}^{d} \times \Omega$.

(A5) $\phi \in L^{\infty}(\Omega)$ and there exist positive real numbers $\phi_{\circ}, \phi^{\circ}$ such that $\phi_{\circ} \leq \phi(x) \leq \phi^{\circ}$.

(A6) $q^{I}, q^{P} \in L^{\infty}\left(0, T ; L^{2}(\Omega)\right)$ satisfy $q^{I}, q^{P} \geq 0$ in $\Omega_{T}$ and

$$
\int_{\Omega} q^{I}(t, x)-q^{P}(t, x) \mathrm{d} x=0
$$

for $t \in(0, T)$;

(A7) $\hat{c} \in L^{\infty}((0, T) \times \Omega)$ and $c_{0} \in L^{\infty}(\Omega)$ satisfy $0 \leq \hat{c}(t, x), c_{0}(x) \leq 1$ in $(0, T) \times \Omega$ and $\Omega$, respectively.

(A8) There exist positive real numbers $\rho_{\circ}, \rho^{\circ}$ such that the Lipschitz continuous function $\rho: \mathbb{R} \rightarrow \mathbb{R}$ satisfies

$$
\rho_{\circ} \leq \rho(c) \leq \rho^{\circ}
$$

for all $c \in \mathbb{R}$. Furthermore, $g$ is a constant vector in $\mathbb{R}^{d}$. 
We denoted here, as throughout the text, the Euclidian norm in $\mathbb{R}^{d}$ by $|\cdot|$. For the sake of simplicity we only consider Neumann boundary conditions. For the formulation of the Dirichlet and the mixed Dirichlet-Neumann problem see [CE99]. The analysis presented in this paper can directly be extended to this setting.

Given a Banach space $X$, let $\mathscr{C}^{i}(0, T ; X)$ be the space of $i$-times continuously differentiable $X$-valued functions on the closed interval $[0, T]$. The space $\mathscr{C}_{c}^{i}(0, T ; X)$ contains the functions which have a compact support in $(0, T)$. Sometimes $\mathscr{C}_{c}^{\infty}(0, T ; X)$ is denoted as $\mathscr{D}(0, T ; X)$ and $\mathscr{C}^{0}(0, T ; X)$ by $\mathscr{C}(0, T ; X)$. The spaces $\mathscr{C}^{i}(0, T ; X)$ and $\mathscr{C}_{c}^{i}(0, T ; X)$ are equipped with the norm of uniform convergence.

We use standard notation for Lebesgue and Sobolev spaces and denote the inner product of $L^{2}(U ; X)$ by $(v, w)_{U}$. The subscript $U$ is skipped if $U=\Omega$ and we abbreviate $\|v\|=\|v\|_{L^{2}(\Omega)}$. The duality pairing between $X$ and its dual space $X^{*}$ is written $\langle\cdot, \cdot\rangle$. Throughout this paper, $C$ denotes a generic constant which may depend on the data but not on mesh size and time step. We often abbreviate $a \leq C b$ by $a \lesssim b$ where $a, b \in \mathbb{R}$.

We set

$$
\begin{aligned}
H_{N}(\operatorname{div} ; \Omega) & :=\left\{v \in L^{2}\left(\Omega ; \mathbb{R}^{d}\right): \operatorname{div} v \in L^{2}(\Omega), v \cdot n=0 \text { in } H^{-1 / 2}(\partial \Omega)\right\} \\
L_{0}^{2}(\Omega) & :=\left\{q \in L^{2}(\Omega): \int_{\Omega} q \mathrm{~d} x=0\right\}
\end{aligned}
$$

Definition 1 (Weak Formulation). A triple

$$
(u, p, c) \in L^{\infty}\left(0, T ; H_{N}(\operatorname{div} ; \Omega)\right) \times L^{\infty}\left(0, T ; L_{0}^{2}(\Omega)\right) \times\left(L^{2}\left(0, T ; H^{1}(\Omega)\right) \cap \mathscr{C}\left(0, T ; W^{1,4}(\Omega)^{*}\right)\right)
$$

is called weak solution of the incompressible miscible flow problem (1) to (5) if

(W1) for $t \in(0, T), v \in H_{N}(\operatorname{div} ; \Omega)$ and $q \in L_{0}^{2}(\Omega)$

$$
\begin{aligned}
\left(\mu(c) \mathbb{K}^{-1} u, v\right)-(p, \operatorname{div} v) & =(\rho(c) g, v) \\
(q, \operatorname{div} u) & =\left(q^{I}-q^{P}, q\right) .
\end{aligned}
$$

(W2) for all $w \in \mathscr{D}\left(0, T ; W^{1,4}(\Omega)\right)$

$$
\int_{0}^{T}-\left(\phi c, \partial_{t} w\right)+(\mathbb{D}(u) \nabla c, \nabla w)+(u \cdot \nabla c, w)+\left(q^{I} c, w\right)-\left(\hat{c} q^{I}, w\right) \mathrm{d} t=0 .
$$

(W3) $c(0, \cdot)=c_{0}$ in $W^{1,4}(\Omega)^{*}$.

Remark 1. We do not discuss the question whether weak solutions satisfy the maximum principle $0 \leq c \leq 1$ almost everywhere in $(0, T) \times \Omega$. The results in [F94, CE99] show that such solutions exist. Therefore, if the weak solution is unique, which holds if $p \in L^{\infty}\left(0, T ; W^{1, \infty}(\Omega)\right)$, then the maximum principle is satisfied. Moreover, one can show directly that weak solutions satisfy the maximum principle if $c \in L^{2}\left(0, T ; W^{1,4}(\Omega)\right)$ or if $\mathbb{D}$ is uniformly bounded.

\section{The Finite Element Spaces}

Let $0=t_{0}<t_{1}<\ldots<t_{M}=T$ be a partition of the time interval $[0, T]$. We define $k_{j}:=t_{j}-t_{j-1}$ and introduce the backward Euler operator $d_{t} a^{j}:=k_{j}^{-1}\left(a^{j}-a^{j-1}\right)$ for $j=1,2, \ldots, M$ and arbitrary sequences $\left(a^{j}\right)_{j=0,1, \ldots, M}$. We set $k:[0, T] \rightarrow \mathbb{R}, t \in\left(t_{j-1}, t_{j}\right] \mapsto k_{j}$.

We consider partitions $\mathcal{T}$ of $\Omega$ which consist of convex, closed polyhedral elements $K$ and set $h_{K}:=\operatorname{diam}(K)$. We define $h_{\mathcal{T}}: \Omega \rightarrow \mathbb{R}$ in the interior of elements by

$$
x \in \operatorname{interior}(K) \Longrightarrow h_{\mathcal{T}}(x)=h_{K}
$$


and fix that $h_{\mathcal{T}}$ attains on internal element faces the diameter of the smaller adjacent element, cf. (M5) below. In other words $h_{\mathcal{T}}$ is the lower semi-continuous function which satisfies (6). The space of polynomials with total degree $p$ is denoted by $\mathcal{P}^{p}$ and the space of tensor polynomials with partial degree $p$ is denoted by $\mathcal{Q}^{p}$. The mapping between $K \in \mathcal{T}$ and its reference element $\hat{K}$ is written $F_{K}: \hat{K} \rightarrow K$. For simplicity we assume that $F_{K}$ is affine. Given $p \in \mathbb{N}$, set

$$
\mathcal{S}^{p}(\mathcal{T}, \mathcal{R}):=\left\{w \in L^{2}(\Omega): w \circ F_{K} \in \mathcal{R}^{p} \forall K \in \mathcal{T}\right\}, \quad \mathcal{R} \in\{\mathcal{P}, \mathcal{Q}\} .
$$

If unambiguous we sometimes write $\mathcal{S}^{p}(\mathcal{T})$ instead of $\mathcal{S}^{p}(\mathcal{T}, \mathcal{R})$.

For $w_{h} \in \mathcal{S}^{p}(\mathcal{T})$ the function $\nabla_{h} w_{h}$ is defined through $\left.\left(\nabla_{h} w_{h}\right)\right|_{K}=\nabla\left(\left.w_{h}\right|_{K}\right)$ for all $K \in \mathcal{T}$, i.e. $\nabla_{h}$ denotes the elementwise application of the gradient operator. As usual the sets of interior and boundary faces are

$$
\begin{aligned}
\mathcal{E}_{\Omega}(\mathcal{T}) & :=\left\{K \cap K^{\prime}: K, K^{\prime} \in \mathcal{T}, K \cap K^{\prime} \text { is } d-1 \text { dimensional }\right\} \\
\mathcal{E}_{\partial \Omega}(\mathcal{T}) & :=\{K \cap \partial \Omega: K \in \mathcal{T}, K \cap \partial \Omega \text { is } d-1 \text { dimensional }\}
\end{aligned}
$$

respectively. We set $\mathcal{E}(\mathcal{T})=\mathcal{E}_{\Omega}(\mathcal{T}) \cup \mathcal{E}_{\partial \Omega}(\mathcal{T})$ and assign to each $E \in \mathcal{E}(\mathcal{T})$ its diameter $h_{E}$. By abuse of language we often also denote $\{x \in \bar{\Omega}: x \in E, E \in \mathcal{E}(\mathcal{T})\}$ by $\mathcal{E}(\mathcal{T})$. Similarly, $\mathcal{E}_{\Omega}(\mathcal{T})$ denotes the set of all interior edges as well as the union of those. The unit outward normal vector of $K \in \mathcal{T}$ is $n_{K}$. We assign to each $E \in \mathcal{E}(\mathcal{T})$ a unit normal vector $n_{E}$. The functions $h_{\mathcal{E}} \in L^{\infty}(\mathcal{E}(\mathcal{T}))$ and $n_{\mathcal{E}} \in L^{\infty}(\mathcal{E}(\mathcal{T}))$ are defined through $\left.h_{\mathcal{E}}\right|_{E}=h_{E}$ and $\left.n_{\mathcal{E}}\right|_{E}=n_{E}$, respectively. Frequently, we denote two neighbouring elements by $K^{+}$and $K^{-}$. Given elementwise smooth functions $v_{h}: \Omega \rightarrow X, X \in\left\{\mathbb{R}, \mathbb{R}^{d}, \mathbb{R}^{d \times d}\right\}$, we set for the edge $E=K^{+} \cap K^{-}$

$$
v^{+}:=\left.\left(\left.v_{h}\right|_{K^{+}}\right)\right|_{E}, \quad v^{-}:=\left.\left(\left.v_{h}\right|_{K^{-}}\right)\right|_{E}
$$

and define the jump and the average of $v_{h}$ by

$$
\left.\left[v_{h}\right]\right|_{E}:=v^{+}-v^{-},\left.\quad\left\{v_{h}\right\}\right|_{E}:=\frac{v^{+}+v^{-}}{2},
$$

respectively. For $E \in \mathcal{E}_{\partial \Omega}(\mathcal{T})$ we define

$$
\left.\left[v_{h}\right]\right|_{E}:=v_{h},\left.\quad\left\{v_{h}\right\}\right|_{E}:=v_{h} .
$$

We use the mesh-dependent norm

$$
\|v\|_{\mathcal{T}}^{2}:=\|v\|^{2}+\left\|\nabla_{h} v\right\|^{2}+\left\|h_{\mathcal{E}}^{-1 / 2}[v]\right\|_{\mathcal{E}_{\Omega}^{j}}^{2} .
$$

The concentration $c$ is discretised with a discontinuous Galerkin method and denote the mesh for $c$ at time $j$ by $\mathcal{T}_{c}^{j}$ or simply by $\mathcal{T}^{j}$. We assume that there are only finitely many reference elements underlying $\mathcal{T}_{c}^{j}$. The approximation space for the variable $c$ at time step $j$ is denoted by $\mathcal{S}_{c}^{j}$. Often we abbreviate $\mathcal{E}^{j}:=\mathcal{E}\left(\mathcal{T}_{c}^{j}\right)$, $\mathcal{E}_{\Omega}^{j}:=\mathcal{E}_{\Omega}\left(\mathcal{T}_{c}^{j}\right), \mathcal{E}_{\partial \Omega}^{j}:=\mathcal{E}_{\partial \Omega}\left(\mathcal{T}_{c}^{j}\right)$.

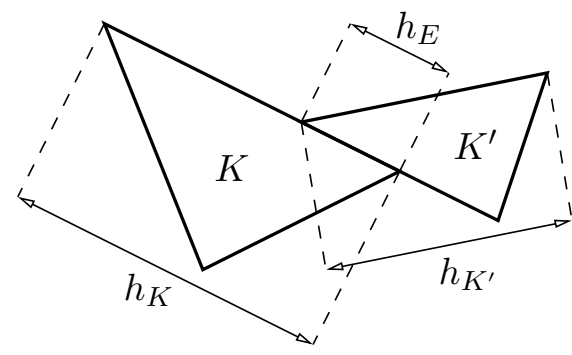

Definition of $h_{K}$ and $h_{E}$. 
The variables $u$ and $p$ are approximated with the mixed Raviart-Thomas finite element method. More specifically, we assume that the mesh $\mathcal{T}_{u}^{j}$, discretising $u$ and $p$ at time $t_{j}$, consists either entirely of triangles or entirely of quadrilaterals if $d=2$ and consists either entirely of tetrahedra or entirely of hexahedra if $d=3$. Moreover, $\mathcal{T}_{u}^{j}$ has no hanging nodes. However, we remark that these assumptions on $\mathcal{T}_{u}^{j}$ are not essential and that the analysis remains valid for alternative discretisations of $u$ and $p$ as long as $u_{h}$ is conforming in $H_{N}(\operatorname{div} ; \Omega)$ and Strang's lemma can be applied in the sense of Theorem 10. Alignment of $\mathcal{T}_{c}^{j}$ and $\mathcal{T}_{u}^{j}$ is not necessary. We introduce

$$
\mathcal{R} T^{\ell}\left(\mathcal{T}_{u}^{j}\right):=\left\{v \in H(\operatorname{div} ; \Omega): v \circ F_{K} \in\left(\mathcal{R}^{\ell}\right)^{d}+x \mathcal{R}^{\ell} \forall K \in \mathcal{T}_{u}^{j}\right\} .
$$

Here $\ell$ is a non-negative integer, uniform at time $t_{j}$. If $\mathcal{T}_{u}^{j}$ consists of triangles or tetrahedra we set $\mathcal{R}^{\ell}=\mathcal{P}^{\ell}$, otherwise $\mathcal{R}^{\ell}=\mathcal{Q}^{\ell}$. The approximation spaces of $u$ and $p$ are

$$
\mathcal{S}_{u}^{j}:=\mathcal{R} T^{\ell}\left(\mathcal{T}_{u}^{j}\right) \cap H_{N}(\operatorname{div} ; \Omega), \quad \mathcal{S}_{p}^{j}:=\mathcal{S}^{\ell}\left(\mathcal{T}_{u}^{j}\right) \cap L_{0}^{2}(\Omega) .
$$

We frequently refer to the global mesh size and time step

$$
h^{j}:=\max _{K \in \mathcal{T}_{c}^{j} \cup \mathcal{T}_{u}^{j}} h_{K}, \quad \widetilde{h}:=\max _{0 \leq j \leq M} h^{j}, \quad \widetilde{k}:=\max _{0 \leq j \leq M} k^{j} .
$$

as well as to

$$
\mathcal{S}_{u}:=\prod_{j=1}^{M} \mathcal{S}_{u}^{j}, \quad \mathcal{S}_{p}:=\prod_{j=1}^{M} \mathcal{S}_{p}^{j}, \quad \mathcal{S}_{c}:=\prod_{j=0}^{M} \mathcal{S}_{c}^{j} .
$$

The finite element discretisation is based on the following main assumptions:

(M1) $\mathcal{T}_{c}^{j}$ and $\mathcal{T}_{u}^{j}$ are shape-regular.

(M2) There is a fixed $p \in \mathbb{N}$ such that $\mathcal{S}^{1}\left(\mathcal{T}_{c}^{j}, \mathcal{P}\right) \subset \mathcal{S}_{c}^{j} \subset \mathcal{S}^{p}\left(\mathcal{T}_{c}^{j}, \mathcal{Q}\right)$.

(M3) There is a fixed $\ell^{\circ} \in \mathbb{N}$ such that $0 \leq \ell \leq \ell^{\circ}$ for all $\mathcal{T}_{u}^{j}$.

(M4) $\|v\|_{L^{4}(\Omega)} \lesssim\|v\|_{\mathcal{T}_{c}^{j}}$ for all $v \in \mathcal{S}^{p}\left(\mathcal{T}_{c}^{j}, \mathcal{Q}\right)$.

(M5) $h_{\mathcal{T}_{c}^{j}} \lesssim h_{\mathcal{E}\left(\mathcal{T}_{c}^{j}\right)}$ on the restriction to $\mathcal{E}\left(\mathcal{T}_{c}^{j}\right)$.

Condition (M2) allows non-uniform and anisotropic p. Condition (M4) is, for example, satisfied if there is a recovery operator $R: \mathcal{S}^{p}(\mathcal{T}, \mathcal{Q}) \rightarrow H^{1}(\Omega)$ such that

$$
\left\|h_{\mathcal{T}}^{-1}(v-R v)\right\|+\|R v\|_{H^{1}(\Omega)} \lesssim\|v\|_{\mathcal{T}} .
$$

For details on such recovery operators we refer, for instance, to [BO07], [KP03] and [BG88] and to the references therein. The bound $h_{\mathcal{T}_{c}}^{j} \lesssim h_{\mathcal{E}\left(\mathcal{T}_{c}\right)}^{j}$ in (M5) is, together with (M4), the only restriction on the use of hanging nodes in $\mathcal{T}_{c}$.

In the course of the analysis another mesh condition, namely (21), will become apparent, which controls how much the spaces $\mathcal{S}_{c}^{j}$ differ from each other for different time steps $t_{j}$.

\section{The Finite Element Method}

In order to deal with discontinuous coefficients and to control the time derivative of $c_{h}$ we project $\mathbb{D}$ onto a space of piecewise polynomial functions:

$$
\mathbb{D}_{h}: L^{2}(\Omega)^{d} \rightarrow \mathcal{S}^{p}\left(\mathcal{T}_{c}, \mathbb{R}^{d \times d}\right), v \mapsto \Pi_{\mathcal{T}} \circ \mathbb{D}(v, \cdot)
$$


where the $\Pi_{\mathcal{T}}: L^{2}(\Omega)_{\text {sym }}^{d \times d} \rightarrow \mathcal{S}^{p}\left(\mathcal{T}_{c}, \mathbb{R}_{\text {sym }}^{d \times d}\right)$ are projections which map pointwise onto symmetric matrices such that $\left\|\Pi_{\mathcal{T}} D\right\|_{K} \lesssim\|D\|_{K}$ for all $D \in L^{2}(\Omega)_{\text {sym }}^{d \times d}$ and $K \in \mathcal{T}$. If $\mathbb{D}$ is elementwise polynomial, as is often the case if $\mathbb{D}$ is aligned with the mesh, then one may choose $\mathbb{D}=\mathbb{D}_{h}$. Otherwise, one can select $\mathbb{D}_{h}$ to be the $L^{2}$-projection of $\mathbb{D}$, covering the case that the evaluation of the trace of $\mathbb{D}$ on element boundaries causes computational or theoretical difficulties.

The diffusion term of the concentration equation is discretised by the symmetric interior penalty discontinuous Galerkin method: Given a $u_{h} \in \mathcal{S}_{u}^{j}$, we set

$$
\begin{aligned}
B_{d}\left(c_{h}, w_{h} ; u_{h}\right):= & \left(\mathbb{D}_{h}\left(u_{h}\right) \nabla_{h} c_{h}, \nabla_{h} w_{h}\right)-\left(n_{\mathcal{E}}\left[c_{h}\right],\left\{\mathbb{D}_{h}\left(u_{h}\right) \nabla_{h} w_{h}\right\}\right)_{\mathcal{E}_{\Omega}^{j}} \\
& -\left(n_{\mathcal{E}}\left[w_{h}\right],\left\{\mathbb{D}_{h}\left(u_{h}\right) \nabla_{h} c_{h}\right\}\right)_{\mathcal{E}_{\Omega}^{j}}+\left(\sigma^{2}\left[c_{h}\right],\left[w_{h}\right]\right)_{\mathcal{E}_{\Omega}^{j}}
\end{aligned}
$$

where

$$
\sigma^{2}: \mathcal{E}_{\Omega}^{j} \rightarrow \mathbb{R}, x \mapsto C_{\sigma} \frac{\max \left\{n_{\mathcal{E}}^{\top} \mathbb{D}_{h}\left(u_{h}^{+}, x\right) n_{\mathcal{E}}, n_{\mathcal{E}}^{\top} \mathbb{D}_{h}\left(u_{h}^{-}, x\right) n_{\mathcal{E}}\right\}}{h_{\mathcal{E}}}
$$

and $C_{\sigma}$ is selected such that

$$
C_{\sigma} \geq \sup \left\{h_{K} \max \left\{\frac{\left\|w_{h}\right\|_{\partial K}^{2}}{\left\|w_{h}\right\|_{K}^{2}}, \frac{\left\|D^{1 / 2} \nabla w_{h}\right\|_{\partial K}^{2}}{\left\|D^{1 / 2} \nabla w_{h}\right\|_{K}^{2}}\right\}: w_{h} \in \mathcal{P}^{p}, D \in\left[\mathcal{P}^{p}\right]^{d \times d}, K \text { shape-regular }\right\} .
$$

From a compactness and scaling argument it follows that $C_{\sigma}$ is a finite number, depending on $p$, the degree of shape-regularity and the type of the finite elements used. Because, in principle, the right-hand side of (9) can be computed explicitly, $B_{d}$ is from the theoretical point of view a parameter-free method.

Denoting edges with the letter $E$, we observe that

$$
\begin{aligned}
\left|\left(n_{\mathcal{E}}\left[c_{h}\right],\left\{\mathbb{D}_{h}\left(u_{h}\right) \nabla w_{h}\right\}\right)_{\mathcal{E}_{\Omega}^{j}}\right| & \leq 1 / 2 \sum_{K \in \mathcal{T}} \sum_{E \subset \partial K}\left\|\mathbb{D}_{h}\left(u_{h}^{+}\right)^{1 / 2} \cdot n_{\mathcal{E}}\left[c_{h}\right]\right\|_{E}\left\|\mathrm{D}_{h}\left(u_{h}^{+}\right)^{1 / 2} \nabla w_{h}^{+}\right\|_{E} \\
& \leq 1 / 2 \sum_{K \in \mathcal{T}}\left\|\mathbb{D}_{h}\left(u_{h}^{+}\right)^{1 / 2} \cdot n_{\mathcal{E}}\left[c_{h}\right] / \sqrt{h_{K}}\right\|_{\partial K}\left\|\mathbb{D}_{h}\left(u_{h}^{+}\right)^{1 / 2} \nabla w_{h}^{+} \sqrt{h_{K}}\right\|_{\partial K} \\
& \leq\left\|\sigma\left[c_{h}\right]\right\|_{\mathcal{E}_{\Omega}^{j}}\left\|\mathrm{D}_{h}\left(u_{h}\right)^{1 / 2} \nabla w_{h}\right\|_{\Omega}
\end{aligned}
$$

With $w_{h}=c_{h}$ one concludes that

$$
B_{d}\left(c_{h}, c_{h} ; u_{h}\right) \geq 1 / 2\left(\mathbb{D}_{h}\left(u_{h}\right) \nabla_{h} c_{h}, \nabla_{h} c_{h}\right)+1 / 2\left(\sigma^{2}\left[c_{h}\right],\left[c_{h}\right]\right)_{\mathcal{E}_{\Omega}^{j}}
$$

The convection, injection and production terms are represented by

$$
\begin{aligned}
B_{c q}\left(c_{h}, w_{h} ; u_{h}\right):=1 / 2 & \left(\left(u_{h} \nabla_{h} c_{h}, w_{h}\right)-\left(u_{h} c_{h}, \nabla_{h} w_{h}\right)+\left(\left(q^{I}+q^{P}\right) c_{h}, w_{h}\right)\right. \\
& \left.+\sum_{K \in \mathcal{T}^{j}}\left(c_{h}^{+},\left(u_{h} \cdot n_{K}\right)_{+}\left[w_{h}\right]\right)_{\partial K \backslash \partial \Omega}-\left(\left(u_{h} \cdot n_{K}\right)_{-}\left[c_{h}\right], w_{h}^{+}\right)_{\partial K \backslash \partial \Omega}\right)
\end{aligned}
$$

where $\left(u_{h} \cdot n\right)_{+}:=\max \left\{u_{h} \cdot n, 0\right\}$ and $\left(u_{h} \cdot n\right)_{-}:=\min \left\{u_{h} \cdot n, 0\right\}$. This formulation of $B_{c q}$ differs from the standard $\mathrm{dG}$ formulation in that it descretises first-order terms half in primal form and half in dual form. Commonly, first order operators are approximated by $\mathrm{dG}$ methods of the type

$$
\left(\widetilde{u} \nabla_{h} c_{h}, w_{h}\right)-\sum_{K \in \mathcal{T}^{j}}\left(\left(\widetilde{u} \cdot n_{K}\right)_{-}\left[c_{h}\right],\left.w_{h}\right|_{K}\right)_{\partial K},
$$

where $\widetilde{u}$ is a coefficient of the differential equation in consideration. One also finds formulations in fully dual form in the literature. The definition of $B_{c q}$ is based on a reformulation of the concentration equation before discretisation:

$$
u \cdot \nabla c=1 / 2 u \cdot \nabla c+1 / 2 \operatorname{div}(u c)-1 / 2 \operatorname{div}(u) c=1 / 2 u \cdot \nabla c+1 / 2 \operatorname{div}(u c)-1 / 2\left(q^{I}-q^{P}\right) c,
$$


using that $\operatorname{div} u=q^{I}-q^{P}$ is known. The advantage of (12) is that $B_{c q}$ is semi-definite regardless of the properties of $\operatorname{div} u_{h}$ :

$$
\begin{aligned}
B_{c q}\left(c_{h}, c_{h} ; u_{h}\right) & =1 / 2\left(\left(q^{I}+q^{P}\right) c_{h}, c_{h}\right)+\sum_{K \in \mathcal{T}^{j}}\left(c_{h}^{+},\left(u_{h} \cdot n_{K}\right)_{+}\left[c_{h}\right]\right)_{\partial K \backslash \partial \Omega}-\left(\left(u_{h} \cdot n_{K}\right)_{-}\left[c_{h}\right], c_{h}^{+}\right)_{\partial K \backslash \partial \Omega} \\
& =1 / 2\left(\left(q^{I}+q^{P}\right) c_{h}, c_{h}\right)+\sum_{K \in \mathcal{T}^{j}}\left(c_{h}^{+},\left(u_{h} \cdot n_{K}\right)_{+}\left[c_{h}\right]\right)_{\partial K \backslash \partial \Omega}-\left(\left(u_{h} \cdot n_{K}\right)_{+}\left[c_{h}\right], c_{h}^{-}\right)_{\partial K \backslash \partial \Omega} \\
& =1 / 2\left(\left(q^{I}+q^{P}\right) c_{h}, c_{h}\right)+1 / 2\left(\left|u_{h} \cdot n_{\mathcal{E}^{j}}\right|\left[c_{h}\right],\left[c_{h}\right]\right)_{\mathcal{E}_{\Omega}^{j}},
\end{aligned}
$$

recalling that $u_{h} \in H_{N}(\operatorname{div} ; \Omega)$ and that therefore $u_{h}^{+} \cdot n_{E}=u_{h}^{-} \cdot n_{E}$ for $E \in \mathcal{E}_{\Omega}^{j}$. Due to (14) the numerical method does not need a regularisation of $u_{h}$ as for example used in [SRW02].

We consider the following method to solve the boundary value problem:

Algorithm $\left(A^{d G}\right)$ Choose $c_{h}^{0} \in \mathcal{S}_{c}^{0}$. For $1 \leq j \leq M$ and $c_{h}^{j-1} \in \mathcal{S}_{c}^{j-1}$ find $\left(u_{h}^{j}, p_{h}^{j}, c_{h}^{j}\right) \in \mathcal{S}_{u}^{j} \times \mathcal{S}_{p}^{j} \times \mathcal{S}_{c}^{j}$ such that

$$
\begin{array}{ll}
\left(\mu\left(c_{h}^{j}\right) \mathbb{K}^{-1} u_{h}^{j}, v_{h}\right)-\left(p_{h}^{j}, \operatorname{div} v_{h}\right) & =\left(\rho\left(c_{h}^{j}\right) g, v_{h}\right), \\
\left(q_{h}, \operatorname{div} u_{h}^{j}\right) & =\left(q^{I}-q^{P}, q_{h}\right)
\end{array}
$$

for all $\left(v_{h}, q_{h}\right) \in \mathcal{S}_{u}^{j} \times \mathcal{S}_{p}^{j}$ and

$$
\left(\phi d_{t} c_{h}^{j}, w_{h}\right)+B_{d}\left(c_{h}^{j}, w_{h} ; u_{h}^{j}\right)+B_{c q}\left(c_{h}^{j}, w_{h} ; u_{h}^{j}\right)=\left(\hat{c} q^{I}, w_{h}\right)
$$

for all $w_{h} \in \mathcal{S}_{c}^{j}$.

Algorithm $\left(A^{d G}\right)$ generates an element $\left(u_{h}, p_{h}, c_{h}\right) \in \mathcal{S}_{u} \times \mathcal{S}_{p} \times \mathcal{S}_{c}$ to approximate $(u, p, c)$.

Remark 2. A delicate issue about the discretisation of the incompressible miscible flow problem is that the natural choice of function spaces gives, in first instance, only $H$ (div)-control on the Darcy velocity and therefore provides direct access only to the scalar field $u \cdot n_{K}$ on element boundaries. In contrast, the diffusive flux $\left.\mathbb{D}(u)\right|_{\partial K}$ is only meaningful if all components of $u$ are known, defining $\mathbb{D}$ for example as in (39). Our analysis shows that (M4) and (M5) as well as the projection onto $\mathbb{D}_{h}$ ensure sufficient control on the diffusive flux to guarantee convergence to a weak solution.

Applying standard techniques [HSS02] [J05] and (15) one can rewrite $B_{c q}$ to resemble the traditional, fully primal formulation (13) more closely:

$$
B_{c q}\left(c_{h}, w_{h} ; u_{h}\right)=\left(u_{h} \nabla_{h} c_{h}, w_{h}\right)+{ }^{1} / 2\left(\left(q^{I}+q_{h}^{I}+q^{P}-q_{h}^{P}\right) c_{h}, w_{h}\right)-\sum_{K \in \mathcal{T}^{j}}\left(\left(u_{h} \cdot n_{K}\right)_{-}\left[c_{h}\right], w_{h}^{+}\right)_{\partial K \backslash \partial \Omega} .
$$

Here $q_{h}^{I}$ and $q_{h}^{P}$ are the $L^{2}$-projections of $q^{I}$ and $q^{P}$ onto $\mathcal{S}_{p}$, respectively. Clearly, if $q^{I}, q^{P} \in \mathcal{S}_{p}$ then $B_{c q}$ takes the form of (13) exactly.

\section{Unconditional Well-posedness and Stability}

The aim of this section is to prove that the solutions to Algorithm $\left(A^{d G}\right)$ exist and that they are bounded independently of the discretisation parameters.

Theorem 1. Given $c_{h}^{j-1} \in \mathcal{S}_{c}^{j-1}$, there exists a solution $\left(u_{h}^{j}, p_{h}^{j}, c_{h}^{j}\right) \in \mathcal{S}_{u}^{j} \times \mathcal{S}_{p}^{j} \times \mathcal{S}_{c}^{j}$ to the simultaneous system of equations (15) and (16).

Proof. Given any $c_{h} \in \mathcal{S}_{c}^{j}$, let $\left(u_{h}, p_{h}\right)$ be the unique pair in $\mathcal{S}_{u}^{j} \times \mathcal{S}_{p}^{j}$ which satisfies

$$
\begin{aligned}
\left(\mu\left(c_{h}\right) \mathbb{K}^{-1} u_{h}, v_{h}\right)-\left(p_{h}, \operatorname{div} v_{h}\right) & =\left(\rho\left(c_{h}\right) g, v_{h}\right), \\
\left(q_{h}, \operatorname{div} u_{h}\right) & =\left(q^{I}-q^{P}, q_{h}\right)
\end{aligned}
$$


for all $\left(v_{h}, q_{h}\right) \in \mathcal{S}_{u}^{j} \times \mathcal{S}_{p}^{j}$. We define the mapping $\Phi: \mathcal{S}_{c}^{j} \rightarrow\left(\mathcal{S}_{c}^{j}\right)^{*}$ as the residual of (16), i.e.

$$
\Phi\left(c_{h}\right)\left[w_{h}\right]:=k_{j}^{-1}\left(\phi\left(c_{h}-c_{h}^{j-1}\right), w_{h}\right)+B_{d}\left(c_{h}, w_{h} ; u_{h}\right)+B_{c q}\left(c_{h}, w_{h} ; u_{h}\right)-\left(\hat{c} q^{I}, w_{h}\right) .
$$

Recalling (11) and (14), the choice $w_{h}=c_{h}$ gives

$$
\begin{aligned}
\Phi\left(c_{h}\right)\left[c_{h}\right]= & \frac{\left\|\phi^{1 / 2} c_{h}\right\|^{2}-\left\|\phi^{1 / 2} c_{h}^{j-1}\right\|^{2}}{2 k_{j}}+\frac{k_{j}}{2}\left\|\frac{\phi^{1 / 2}}{k_{j}}\left(c_{h}-c_{h}^{j-1}\right)\right\|^{2} \\
& +B_{d}\left(c_{h}, c_{h} ; u_{h}\right)+B_{c q}\left(c_{h}, c_{h} ; u_{h}\right)-\left(\hat{c} q^{I}, c_{h}\right) \\
\geq & \frac{1}{2 k_{j}}\left(\left\|\phi^{1 / 2} c_{h}\right\|^{2}-\left\|\phi^{1 / 2} c_{h}^{j-1}\right\|^{2}\right)-{ }^{1 / 2}\left\|\left(q^{I}\right)^{1 / 2} \hat{c}_{h}\right\|^{2} .
\end{aligned}
$$

Therefore, $\Phi\left(c_{h}\right)\left[c_{h}\right] \geq 0$ for all $c_{h} \in \mathcal{S}_{c}^{j}$ such that

$$
\left\|\phi^{1 / 2} c_{h}\right\|^{2} \geq\left\|\phi^{1 / 2} c_{h}^{j-1}\right\|^{2}+k_{j}\left\|\left(q^{I}\right)^{1 / 2} \hat{c}_{h}^{j}\right\|^{2} .
$$

The existence of $c_{h}^{j} \in \mathcal{S}_{c}^{j}$ with $\Phi\left(c_{h}^{j}\right)=0$ is now a corollary of Brouwer's fixed point theorem, e.g. [Z1, p. 54]. This $c_{h}^{j}$ solves (16).

In the subsequent text we frequently use the piecewise linear interpolant of $c_{h}$. For $t \in\left[t_{j-1}, t_{j}\right]$, let

$$
\widetilde{c}_{h}(t, \cdot):=\frac{t-t_{j-1}}{k_{j}} c_{h}^{j}+\frac{t_{j}-t}{k_{j}} c_{h}^{j-1} .
$$

Observe that $\partial_{t} \widetilde{c}_{h}(t, \cdot)=d_{t} c_{h}^{j}(\cdot), t \in\left(t_{j-1}, t_{j}\right)$. We shall also interpret functions in $\mathcal{S}_{u}, \mathcal{S}_{p}$ and $\mathcal{S}_{c}$ as timedependent functions by assuming they attain on the intervals $\left(t_{j-1}, t_{j}\right]$ a constant value in $\mathcal{S}_{u}^{j}$, $\mathcal{S}_{p}^{j}$ and $\mathcal{S}_{c}^{j}$, respectively. We define the spatial semi-norm

$$
\left|c_{h}\right|_{u_{h}}^{2}:=\left(\mathbb{D}_{h}\left(u_{h}\right) \nabla_{h} c_{h}, \nabla_{h} c_{h}\right)+\left(\sigma^{2}\left[c_{h}\right],\left[c_{h}\right]\right)_{\mathcal{E}_{\Omega}^{j}}+\left(\left|u_{h} \cdot n_{\mathcal{E}^{j}}\right|\left[c_{h}\right],\left[c_{h}\right]\right)_{\mathcal{E}_{\Omega}^{j}}
$$

Theorem 2. There exists a constant $C>0$ such that

$$
\left\|u_{h}^{j}\right\|+\left\|\operatorname{div} u_{h}^{j}\right\|+\left\|p_{h}^{j}\right\| \lesssim\left(\left\|\rho^{\circ} g\right\|+\left\|q^{I}-q^{P}\right\|\right)
$$

holds for all $j=1,2, \ldots, M$. Equally we have

$$
\left\|\phi^{1 / 2} c_{h}^{j}\right\|^{2}+\int_{0}^{t_{j}} k\left\|\phi^{1 / 2} \partial_{t} \widetilde{c}_{h}\right\|^{2}+\left|c_{h}\right|_{u_{h}^{j}}^{2} \mathrm{~d} t \leq\left\|\phi^{1 / 2} c_{h}^{0}\right\|^{2}+\int_{0}^{t_{j}}\left\|\sqrt{q^{I}} \hat{c}\right\|^{2} \mathrm{~d} t
$$

for all $j=1,2, \ldots, M$.

Proof. The first assertion is a consequence of the inf-sup condition, which is satisfied by the Raviart-Thomas mixed finite element method for elliptic equations [BF91, p. 138]. To prove the second assertion we choose $w_{h}=c_{h}^{i}$ in (16) to verify that

$$
\begin{aligned}
& d_{t}\left\|\phi^{1 / 2} c_{h}^{i}\right\|^{2}+k_{i}\left\|\phi^{1 / 2} d_{t} c_{h}^{i}\right\|^{2}+\left|c_{h}^{i}\right|_{u_{h}^{i}}^{2}+\left\|\sqrt{q^{I}+q^{P}} c_{h}^{i}\right\|^{2} \\
\leq & 2\left(\phi d_{t} c_{h}^{i}, c_{h}^{i}\right)+2 B_{d}\left(c_{h}^{i}, c_{h}^{i} ; u_{h}^{i}\right)+2 B_{c q}\left(c_{h}^{i}, c_{h}^{i} ; u_{h}^{i}\right) \\
= & 2\left(\hat{c} q^{I}, c_{h}^{i}\right) \leq\left\|\sqrt{q^{I}} \hat{c}\right\|^{2}+\left\|\sqrt{q^{I}} c_{h}^{i}\right\|^{2} .
\end{aligned}
$$

Multiplication of the estimate by $k_{i}$ and summation over $i=1,2, \ldots, j$ give

$$
\left\|\phi^{1 / 2} c_{h}^{j}\right\|^{2}+\sum_{i=1}^{j} k_{i}\left(k_{i}\left\|\phi^{1 / 2} d_{t} c_{h}^{i}\right\|^{2}+\left|c_{h}^{i}\right|_{u_{h}^{i}}^{2}\right) \leq\left\|\phi^{1 / 2} c_{h}^{0}\right\|^{2}+\sum_{i=1}^{j} k_{i}\left\|\sqrt{q^{I}} \hat{c}\right\|^{2}
$$

for all $j=1,2, \ldots, M$. 
We record an approximation result of the weighted $L^{2}$-projection $P_{\mathcal{T}}: L^{2}(\Omega) \rightarrow \mathcal{S}^{1}(\mathcal{T}, \mathcal{P})$ which is defined by

$$
(\phi w, v)=\left(\phi P_{\mathcal{T}} w, v\right), \quad v \in \mathcal{S}^{1}(\mathcal{T}, \mathcal{P}) .
$$

We often abbreviate $P_{j}:=P_{\mathcal{T}_{c}^{j}}$. Let $q \in[2, \infty]$ and $w \in W^{r+1, q}(\Omega)$. Then $\xi=w-P_{\mathcal{T}} w$ satisfies

$$
\left\|h_{\mathcal{T}}^{-r-1} \xi\right\|_{L^{q}(\Omega)}+\left\|h_{\mathcal{T}}^{-r} \nabla_{h} \xi\right\|_{L^{q}(\Omega)}+\left(\sum_{K \in \mathcal{T}} h_{K}^{-2 r-1}\|\xi\|_{L^{2}(\partial K)}^{2}+h_{K}^{-2 r+1}\left\|\nabla_{h} \xi\right\|_{L^{2}(\partial K)}^{2}\right)^{1 / 2} \lesssim|w|_{W^{r+1, q}(\Omega)} .
$$

The bound follows from the properties of averaged Taylor polynomials, the Bramble-Hilbert lemma and the trace inequality (28). We refer the reader for details to [BS02, ch. 4].

To analyse the stability in the time derivative we introduce a parameter which measures the effects of coarsening in mesh $\mathcal{T}^{j}$ with respect to $\mathcal{T}^{j-1}$ :

$$
\tau:=\sup \left\{\left(\sum_{j=1}^{M} \int_{t_{j-1}}^{t_{j}} \frac{1}{k_{j}}\left\|P_{j-1}\left(\mathrm{Id}-P_{j}\right) w(t, \cdot)\right\|^{2} \mathrm{~d} t\right)^{1 / 2}:\|w\|_{L^{2}\left(0, T ; W^{P+1,4}(\Omega)\right)} \leq 1\right\} .
$$

The integer $p$ was defined in (M2). If $\mathcal{S}_{c}^{j}$ is the same for all time steps then $P_{j-1}\left(w-P_{j} w\right)=0$ and therefore $\tau=0$. More generally the bound

$$
\tau \lesssim \sup _{j} \sup \left\{h_{K}^{2} / k_{j}: K \in \mathcal{T}_{c}^{j}, K \notin \mathcal{T}_{c}^{j-1}\right\}
$$

is satisfied because

$$
\begin{aligned}
\sum_{j=1}^{M} \int_{t_{j-1}}^{t_{j}} \frac{\left\|P_{j-1}\left(w-P_{j} w\right)\right\|^{2}}{k_{j}} \mathrm{~d} t \lesssim \sum_{j=1}^{M} \int_{t_{j-1}}^{t_{j}} \sum_{K} \frac{\left\|w-P_{j} w\right\|_{K}^{2}}{k_{j}} \mathrm{~d} t \\
\lesssim \sum_{j=1}^{M} \int_{t_{j-1}}^{t_{j}} \sum_{K} \frac{h_{K}^{2}\|w\|_{H^{2}(K)}^{2}}{k_{j}} \mathrm{~d} t \leq\left(\sup _{j, K} h_{K}^{2} / k_{j}\right)\|w\|_{L^{2}\left(0, T ; H^{2}(\Omega)\right)}^{2},
\end{aligned}
$$

where $K$ ranges over all elements in $\mathcal{T}_{c}^{j}$ which are not contained in $\mathcal{T}_{c}^{j-1}$. The right-hand side in (22) can be significantly refined by considering the polynomial degrees $p(K)$ of elements $K$ individually:

$$
\left.\sum_{j=1}^{M} \int_{t_{j-1}}^{t_{j}} \frac{\left\|P_{j-1}\left(w-P_{j} w\right)\right\|^{2}}{k_{j}} \mathrm{~d} t \lesssim\left(\sup _{j, K} h_{K}^{p(K)} / k_{j}\right)\|w\|_{L^{2}(0, T ; W P+1,4}^{2}(\Omega)\right),
$$

Theorem 3. The time derivative $\partial_{t} \widetilde{c}_{h}$ belongs to $L^{2}\left(0, T ; W^{p+1,4}(\Omega)^{*}\right)$ and

$$
\left\|\partial_{t} \widetilde{c}_{h}\right\|_{L^{2}\left(0, T ; W^{P+1,4}(\Omega)^{*}\right)}=\left\|d_{t} c_{h}\right\|_{L^{2}\left(0, T ; W^{p+1,4}(\Omega)^{*}\right)} \lesssim 1+\tau,
$$

independently of the mesh size, time step and polynomial degree.

Proof. Let $w_{h} \in \mathcal{S}_{c}^{j}$. It follows from (10) that

$$
\begin{aligned}
B_{d}\left(c_{h}^{j}, w_{h} ; u_{h}^{j}\right) \lesssim & \left\|\mathbb{D}_{h}\left(u_{h}^{j}\right)^{1 / 2} \nabla_{h} c_{h}^{j}\right\|\left\|\left|u_{h}^{j}\right|^{1 / 2}\right\|_{L^{4}(\Omega)}\left\|\nabla_{h} w_{h}\right\|_{L^{4}(\Omega)}+\left\|\nabla_{h} c_{h}^{j}\right\|\left\|\sigma\left[w_{h}\right]\right\|_{\mathcal{E}_{\Omega}^{j}} \\
& +\left\|\sigma\left[c_{h}^{j}\right]\right\|_{\mathcal{E}_{\Omega}^{j}}\left\|\nabla_{h} w_{h}\right\|+\left\|\sigma\left[c_{h}^{j}\right]\right\|_{\mathcal{E}_{\Omega}^{j}}\left\|\sigma\left[w_{h}\right]\right\|_{\mathcal{E}_{\Omega}^{j}} \\
\lesssim & \left(1+\left\|u_{h}^{j}\right\|^{1 / 2}\right)\left\|c_{h}^{j}\right\|_{\mathcal{T}^{j}}\left(\left\|\nabla_{h} w_{h}\right\|_{L^{4}(\Omega)}+\left\|w_{h}\right\|_{L^{4}(\Omega)}+\left\|\sigma\left[w_{h}\right]\right\|_{\mathcal{E}_{\Omega}^{j}}\right) .
\end{aligned}
$$

Owing to (A4) and (M4) and

$$
\begin{aligned}
\sum_{K \in \mathcal{T}^{j}}\left(\left(c_{h}^{j}\right)^{+},\left(u_{h} \cdot n_{K}\right)_{+}\left[w_{h}\right]\right)_{\partial K \backslash \partial \Omega} & \lesssim \sum_{K \in \mathcal{T}^{j}} h_{K}^{1 / 2}\left\|\mathbb{D}_{h}\left(u_{h}^{+}\right)^{1 / 2}\left(c_{h}^{j}\right)^{+}\right\|_{\partial K \backslash \partial \Omega}\left\|\sigma\left[w_{h}\right]\right\|_{\partial K \backslash \partial \Omega} \\
& \lesssim \sum_{K \in \mathcal{T}^{j}}\left\|\mathbb{D}_{h}\left(u_{h}\right)^{1 / 2} c_{h}^{j}\right\|_{K}\left\|\sigma\left[w_{h}\right]\right\|_{\partial K \backslash \partial \Omega} \\
& \leq\left\|\mathbb{D}_{h}\left(u_{h}\right)\right\|^{1 / 2}\left\|c_{h}^{j}\right\|_{L^{4}(\Omega)}\left\|\sigma\left[w_{h}\right]\right\|_{\mathcal{E}_{\Omega}^{j}},
\end{aligned}
$$


one finds

$$
\begin{aligned}
& 2 B_{c q}\left(c_{h}^{j}, w_{h} ; u_{h}^{j}\right) \\
\lesssim & \left\|\mathbb{D}_{h}\left(u_{h}^{j}\right)^{1 / 2} \nabla_{h} c_{h}^{j}\right\|\left\|\left|u_{h}^{j}\right|^{1 / 2}\right\|_{L^{4}(\Omega)}\left\|w_{h}\right\|_{L^{4}(\Omega)}+\left\|u_{h}^{j}\right\|_{L^{2}(\Omega)}\left\|c_{h}^{j}\right\|_{L^{4}(\Omega)}\left\|\nabla_{h} w_{h}\right\|_{L^{4}(\Omega)} \\
& +\left\|\left(q^{I}+q^{P}\right)^{1 / 2} c_{h}^{j}\right\|\left\|\left(q^{I}+q^{P}\right)^{1 / 2} w_{h}\right\|+\left\|c_{h}^{j}\right\|_{L^{4}(\Omega)}\left\|\sigma\left[w_{h}\right]\right\|_{\mathcal{E}_{\Omega}^{j}}+\left\|w_{h}\right\|_{L^{4}(\Omega)}\left\|\sigma\left[c_{h}^{j}\right]\right\|_{\mathcal{E}_{\Omega}^{j}} \\
\lesssim & \left(1+\left\|u_{h}^{j}\right\|^{1 / 2}\right)\left\|c_{h}^{j}\right\|_{\mathcal{T}^{j}}\left(\left\|\nabla_{h} w_{h}\right\|+\left\|w_{h}\right\|_{L^{4}(\Omega)}+\left\|\sigma\left[w_{h}\right]\right\|_{\mathcal{E}_{\Omega}^{j}}\right) .
\end{aligned}
$$

The Nemyckii trick [Z2, p. 39] and (A4) show that $\mathbb{D}$ and $\mathbb{D}_{h}=\Pi_{\mathcal{T}} \circ \mathbb{D}$ are uniformly bounded operators from $L^{2}(K)^{d \times d}$ to $L^{2}(K)^{d \times d}$ for all $K \in \mathcal{T}_{c}^{j}$. We obtain for $E \in \mathcal{E}_{c}^{j}$ and an adjacent element $K \in \mathcal{T}_{c}^{j}$

$$
\left\|\mathbb{D}_{h}\left(u_{h}^{j}\right)^{+}\right\|_{L^{\infty}(E)} \leq\left\|\mathbb{D}_{h}\left(u_{h}^{j}\right)\right\|_{L^{\infty}(K)} \lesssim h_{K}^{-\frac{d}{2}}\left\|\mathbb{D}_{h}\left(u_{h}^{j}\right)\right\|_{L^{2}(K)} \lesssim h_{K}^{-\frac{d}{2}}\left(1+\left\|u_{h}^{j}\right\|_{K}\right) .
$$

Fix $t \in\left(t_{j-1}, t_{j}\right]$ and $w \in \mathscr{C}_{c}^{\infty}\left(0, T ; \mathscr{C}^{\infty}(\Omega)\right)$. We set $w_{h}(t, \cdot):=P_{j} w(t, \cdot) \in \mathcal{S}_{c}^{j}$. It follows from (M5) that

$$
\begin{aligned}
\left\|\sigma\left[w_{h}\right]\right\|_{\mathcal{E}_{\Omega}^{j}}^{2} & =\left\|\sigma\left[w-w_{h}\right]\right\|_{\mathcal{E}_{\Omega}^{j}}^{2} \lesssim \sum_{E \in \mathcal{E}_{\Omega}^{j}} h_{E}^{-1}\left(\left\|\mathbb{D}_{h}\left(u_{h}^{j}\right)^{+}\right\|_{L^{\infty}(E)}+\left\|\mathbb{D}_{h}\left(u_{h}^{j}\right)^{-}\right\|_{L^{\infty}(E)}\right)\left\|\left[w-w_{h}\right]\right\|_{E}^{2} \\
& \lesssim\left(1+\left\|u_{h}^{j}\right\|\right) \sum_{K \in \mathcal{T}_{c}^{j}} h_{\mathcal{T}_{c}}^{2-d / 2}\|w\|_{H^{2}(K)}^{2} \lesssim\left(1+\left\|u_{h}^{j}\right\|\right) \widetilde{h}^{1 / 2}\|w\|_{H^{2}(\Omega)}^{2} .
\end{aligned}
$$

Note that,

$$
\int_{0}^{T}\left(\phi d_{t} c_{h}^{j}, w\right) \mathrm{d} t=\int_{0}^{T}\left(\phi d_{t} c_{h}^{j}, w_{h}\right) \mathrm{d} t+\int_{0}^{T}\left(\phi d_{t} c_{h}^{j}, w-w_{h}\right) \mathrm{d} t
$$

Using orthogonality and the previous theorem, one concludes

$$
\begin{aligned}
\int_{0}^{T}\left(\phi d_{t} c_{h}^{j}, w-w_{h}\right) \mathrm{d} t & \lesssim\left(\int_{0}^{T} k\left\|\sqrt{\phi} \partial_{t} \widetilde{c}_{h}\right\|^{2} \mathrm{~d} t\right)^{1 / 2}\left(\sum_{j=1}^{M} \int_{t_{j-1}}^{t_{j}} \frac{1}{k_{j}}\left\|\sqrt{\phi} P_{j-1}\left(w-P_{j} w\right)\right\|^{2} \mathrm{~d} t\right)^{1 / 2} \\
& \lesssim \tau\|w\|_{L^{2}\left(0, T ; W^{P+1,4}(\Omega)\right)} .
\end{aligned}
$$

We now turn to the first term on the right-hand side of (25):

$$
\begin{aligned}
\int_{0}^{T}\left(\phi d_{t} c_{h}^{j}, w_{h}\right) \mathrm{d} t & =\int_{0}^{T}-B_{d}\left(c_{h}^{j}, w_{h} ; u_{h}^{j}\right)-B_{c q}\left(c_{h}^{j}, w_{h} ; u_{h}^{j}\right)+\left(\hat{c} q^{I}, w_{h}\right) \mathrm{d} t \\
& \lesssim \int_{0}^{T}\left(1+\left\|u_{h}^{j}\right\|\right)\left(1+\left\|u_{h}^{j}\right\|_{H(\operatorname{div} ; \Omega)}^{1 / 2}\right)\left\|c_{h}^{j}\right\|_{\mathcal{T}^{j}}\|w\|_{W^{P+1,4}(\Omega)} \mathrm{d} t \lesssim\|w\|_{L^{2}\left(0, T ; W^{P+1,4}(\Omega)\right)}
\end{aligned}
$$

using (20). Finally,

$$
\left\|d_{t} c_{h}^{j}\right\|_{L^{2}\left(0, T ; W^{p+1,4}(\Omega)^{*}\right)}=\sup _{w} \frac{\int_{0}^{T}\left(d_{t} c_{h}^{j}, w\right) \mathrm{d} t}{\|w\|_{L^{2}\left(0, T ; W^{p+1,4}(\Omega)\right)}} \lesssim \sup _{w} \frac{\int_{0}^{T}\left(\phi d_{t} c_{h}^{j}, w\right) \mathrm{d} t}{\|w\|_{L^{2}\left(0, T ; W^{p+1,4}(\Omega)\right)}}
$$

completes the proof.

\section{Properties of the Approximation Spaces}

In this section we highlight general properties of discontinuous Galerkin approximation spaces. In particular, the following assumptions are actively used: (M1), (M2) and via (M4) compact embedding into $L^{2}(\Omega)$.

The first theorem shows that $\|\cdot\|_{\mathcal{T}}$-bounded sequences of $\mathrm{dG}$ functions have converging subsequences and that the limits of those subsequences do not have jumps. The statement is independent of the underlying differential equation and numerical method. 
Theorem 4. Consider a sequence $\left(v_{i}\right)_{i}$ such that $v_{i} \in \mathcal{S}^{p}\left(\mathcal{T}_{i}\right)$ and $\left\|v_{i}\right\|_{\mathcal{T}_{i}}<C_{*}$ for some $C_{*}>0$. If $\widetilde{h} \rightarrow 0$ as $i \rightarrow \infty$ then every weak accumulation point of $\left(v_{i}\right)_{i}$ in $L^{2}(\Omega)$ belongs to $H^{1}(\Omega)$. Moreover $\|v\|_{H^{1}(\Omega)} \lesssim C_{*}$ and a subsequence of distributional gradients $\nabla \bar{v}_{i}$ converges weakly in $H^{-1}(\Omega)$ to $\nabla v$.

Proof. Note that the sequence $\left(v^{i}\right)_{i}$ is bounded in $L^{2}(\Omega)$ and therefore admits a weakly converging subsequence in $L^{2}(\Omega)$. For $w \in \mathscr{C}_{c}^{\infty}(\Omega)^{d}$,

$$
\begin{aligned}
\left(\left[v_{i}\right], n_{\mathcal{E}_{i}} \cdot w\right)_{\mathcal{E}_{i}} & \leq\left\|h_{\mathcal{E}_{i}}^{-1 / 2}\left[v_{i}\right]\right\|_{\mathcal{E}_{i}}\left\|h_{\mathcal{E}_{i}}^{1 / 2} w\right\|_{\mathcal{E}_{i}} \leq C_{*}\left\|h_{\mathcal{E}}^{1 / 2} w\right\|_{\mathcal{E}_{i}} \leq C_{*}\left(\sum_{K \in \mathcal{T}_{i}} h_{K}\|w\|_{\partial K}^{2}\right)^{1 / 2} \\
& \lesssim C_{*}\left(\sum_{K \in \mathcal{T}_{i}}\|w\|_{L^{2}(K)}^{2}+h_{K}\|w\|_{L^{2}(K)}|w|_{H^{1}(K)}\right)^{1 / 2} \lesssim C_{*}\left(\|w\|+\widetilde{h}|w|_{H^{1}(\Omega)}\right),
\end{aligned}
$$

using [BS02, p. 39]

$$
\|w\|_{L^{2}(\partial K)}^{2} \leq h_{K}^{-1}\|w\|_{L^{2}(K)}^{2}+\|w\|_{L^{2}(K)}|w|_{H^{1}(K)} .
$$

Therefore the distributional gradient of $v_{i}$ admits the bound

$$
\left|\left(v_{i}, \operatorname{div} w\right)\right|=\left|\left(\nabla_{h} v_{i}, w\right)-\left(\left[v_{i}\right], n_{\mathcal{E}} \cdot w\right)_{\mathcal{E}_{i}}\right| \lesssim C_{*}\left(\|w\|+\widetilde{h}|w|_{H^{1}(\Omega)}\right)
$$

for $w \in \mathscr{C}_{c}^{\infty}(\Omega)^{d}$. Let $v$ be a weak limit of a subsequence $\left(\bar{v}_{i}\right)_{i}$ in $L^{2}(\Omega)$. Then (29) implies that $\nabla v_{i}$ is bounded in $H^{-1}(\Omega)$, giving the weak convergence of the gradient. Furthermore,

$$
|(v, \operatorname{div} w)|=\lim _{i \rightarrow \infty}\left|\left(v_{i}, \operatorname{div} w\right)\right| \lesssim C_{*}\|w\|+\varepsilon
$$

for any $\varepsilon>0$. Therefore $\nabla v \in L^{2}(\Omega)^{d}$ with $\|v\|_{H^{1}(\Omega)} \lesssim C_{*}$.

The proof of Theorem 4 shows that $\|\cdot\|_{\mathcal{T}}$ takes a special role among the norms

$$
\|w\|^{2}+\left\|\nabla_{h} w\right\|^{2}+\left\|h_{\mathcal{E}}^{s}[w]\right\|_{\mathcal{E}_{\Omega}^{j}}^{2}, \quad s \in \mathbb{R} .
$$

On the one hand weak accumulation points $v$ of bounded sequences are only guaranteed to be in $H^{1}(\Omega)$ if $s \leq-1 / 2$. On the other hand the increased flexibility that the gradient $\nabla v$ is composed $\lim _{i}\left(\nabla_{h} v_{i}, \cdot\right)$ and a non-zero part $\lim _{i} \sum_{E}\left(\left[v_{i}\right] \cdot n_{\mathcal{E}},\right)_{E}$ is only seen if $s \geq-1 / 2$. From that point of view it appears possible that the larger approximation space of the $\mathrm{dG}$ method compared to a conforming space can play a qualitatively more significant role in the limit if jumps are not stronger penalised than with order $-1 / 2$.

Example 1. Consider the functions $v_{i}:(0,1) \rightarrow \mathbb{R}$ which are equal to $x \mapsto x$ on $\left(0,{ }^{1} / i\right)$ and which are $1 /{ }_{i}$-periodically extended to $\mathbb{R}$. It is easily checked that $\left\|v_{i}\right\|_{\mathcal{T}_{i}}$ is bounded independently of $i$.

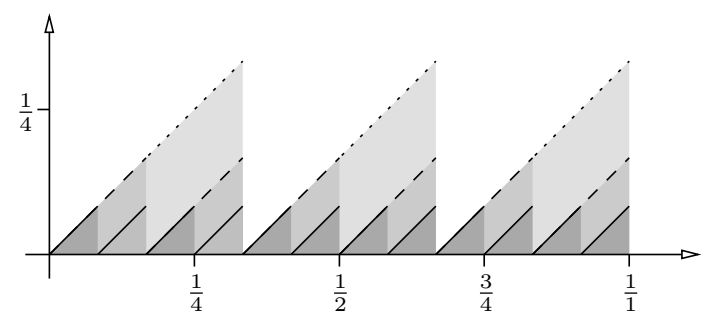

The functions $v_{3}(\cdots), v_{6}(--)$ and $v_{12}(-)$.

The sequence $\left(v_{i}\right)_{i}$ converges to 0 in $L^{2}(0,1)$. Furthermore, $\nabla_{h} v_{i} \equiv 1$ for all $i$, meaning that $\lim _{i} \nabla_{h} v_{i} \equiv 1$. From Theorem 4 we know that the gradient of $\lim _{i} v_{i}$, which is 0 , is the sum of $\lim _{i} \nabla_{h} v_{i}$ and $\lim _{i}-\sum_{E}\left(\left[v_{i}\right]\right.$. $\left.n_{\mathcal{E}}, \cdot\right)_{E}$, implying $\lim _{i}-\sum_{E}\left(\left[v_{i}\right] \cdot n_{\mathcal{E}}, \cdot\right)_{E} \equiv-1$ in the sense of $H^{-1}$. In one space dimension edges $E$ correspond to points in $\mathbb{R}$. 
The following theorem describes accumulation points of $\mathrm{dG}$ sequences in a time-dependent setting.

Theorem 5. Let $\left(\mathcal{S}_{c, i}\right)_{i}=\left(\prod_{j} \mathcal{S}_{c, i}^{j}\right)_{i}$ be a sequence of $d G$ approximation spaces and let $v_{i} \in \mathcal{S}_{c, i}$ such that

$$
\sum_{j=1}^{M} \int_{t_{j-1}}^{t_{j}}\left\|v_{i}(t, \cdot)\right\|_{\mathcal{T}_{i}^{j}}^{2} \mathrm{~d} t<C_{*}
$$

for some $C_{*}>0, i \in \mathbb{N}$. Then there exists a subsequence $\left(\bar{v}_{i}\right)_{i}$ which converges weakly in $L^{2}\left(\Omega_{T}\right)$. If $\widetilde{h} \rightarrow 0$ as $i \rightarrow \infty$ then every weak accumulation point in $L^{2}\left(\Omega_{T}\right)$ belongs to $L^{2}\left(0, T ; H^{1}(\Omega)\right)$. Moreover $\|v\|_{L^{2}\left(0, T ; H^{1}(\Omega)\right)} \lesssim C_{*}$ and a subsequence of distributional gradients $\nabla \bar{v}_{i}$ converges weakly in $L^{2}\left(0, T ; H^{-1}(\Omega)\right)$ to $\nabla v$.

Proof. Observing that for $w \in \mathscr{C}_{c}^{\infty}\left(\Omega_{T}\right)$

$$
\int_{0}^{T}|(v, \operatorname{div} w)| \mathrm{d} t \lesssim \lim _{i \rightarrow \infty} \sum_{j=1}^{M} \int_{t_{j-1}}^{t_{j}}\left\|v_{i}(t, \cdot)\right\|_{\mathcal{T}_{i}^{j}}\left(\|w\|+\widetilde{h}|w|_{H^{1}(\Omega)}\right) \mathrm{d} t \leq C_{*} \int_{0}^{T}\|w\|^{2} \mathrm{~d} t+\varepsilon
$$

the proof is analogous to that of Theorem 4 .

To verify the convergence of the finite element method to a weak solution we require a stronger limit of the gradient than the weak convergence in $H^{-1}(\Omega)$ provided by Theorems 4 and 5 . We define the approximate gradient

$$
G: \mathcal{S}^{p}(\mathcal{T}) \rightarrow \mathcal{S}^{2 p}(\mathcal{T})^{d}, v \mapsto G v
$$

by the condition

$$
\left(\nabla_{h} v, w\right)-\left([v], n_{\mathcal{E}} \cdot\{w\}\right)_{\mathcal{E}(\mathcal{T})}=(G v, w), \quad \forall w \in \mathcal{S}^{2 p}(\mathcal{T})^{d} .
$$

Theorem 6. Let $\left(\mathcal{S}_{c, i}\right)_{i}=\left(\prod_{j} \mathcal{S}_{c, i}^{j}\right)_{i}$ be a sequence of $d G$ approximation spaces and let $v_{i} \in \mathcal{S}_{c, i}$ satisfy (31) for some $C_{*}>0$. If $\widetilde{h} \rightarrow 0$ as $i \rightarrow \infty$ then there exists a subsequence $\left(\bar{v}_{i}\right)_{i}$ which weakly converges in $L^{2}\left(\Omega_{T}\right)$ to a function $v \in L^{2}\left(0, T ; H^{1}(\Omega)\right)$ such that $G \bar{v}_{i}$ converges weakly to $\nabla v$ in $L^{2}\left(\Omega_{T}\right)^{d}$.

Proof. Using the definition of $G$, an argument analogous to (27) and an elementwise inverse inequality we obtain

$$
\begin{aligned}
\left\|G v_{i}\right\|_{\Omega_{T}}^{2} & =\int_{0}^{T}\left(\nabla_{h} v, G v_{i}\right)-\left([v], n_{\mathcal{E}_{i}} \cdot\left\{G v_{i}\right\}\right)_{\mathcal{E}_{i}} \mathrm{~d} t \\
& \leq \int_{0}^{T}\left\|\nabla_{h} v\right\|\left\|G v_{i}\right\|+\left\|h_{\mathcal{E}_{i}}^{-1 / 2}\left[v_{i}\right]\right\|_{\mathcal{E}_{i}}\left(\left\|G v_{i}\right\|+\left(\sum_{T \in \mathcal{T}_{i}}\left|h_{T} G v_{i}\right|_{H^{1}(T)}^{2}\right)^{1 / 2}\right) \mathrm{d} t \lesssim C_{*}\left\|G v_{i}\right\|_{\Omega_{T}} .
\end{aligned}
$$

Division by $\left\|G v_{i}\right\|_{\Omega_{T}}$ gives the boundedness of the sequence. Let $w \in \mathscr{C}_{0}^{\infty}(\Omega)^{d}$. Then, according to Theorem 4 and $(20)$, there is a subsequence $\left(\bar{v}_{i}\right)_{i}$ such that

$$
\begin{aligned}
& \int_{0}^{T}(\nabla v, w) \mathrm{d} t=\lim _{i \rightarrow \infty} \int_{0}^{T}\left(\left(\nabla_{h} \bar{v}_{i}, w\right)-\left(\left[\bar{v}_{i}\right], n_{\mathcal{E}} \cdot w\right)_{\mathcal{E}}\right) \mathrm{d} t \\
= & \lim _{i \rightarrow \infty} \int_{0}^{T}\left(\left(\nabla_{h} \bar{v}_{i}, P_{\mathcal{T}_{i}} w\right)-\left(\left[\bar{v}_{i}\right], n_{\mathcal{E}} \cdot\left\{P_{\mathcal{T}_{i}} w\right\}\right)_{\mathcal{E}}+\left(\nabla_{h} \bar{v}_{i},\left(w-P_{\mathcal{T}_{i}} w\right)\right)-\left(\left[\bar{v}_{i}\right], n_{\mathcal{E}} \cdot\left\{w-P_{\mathcal{T}_{i}} w\right\}\right)_{\mathcal{E}}\right) \mathrm{d} t \\
= & \lim _{i \rightarrow \infty} \int_{0}^{T}\left(\left(G \bar{v}_{i}, P_{\mathcal{T}_{i}} w\right)+\left(\nabla_{h} \bar{v}_{i},\left(w-P_{\mathcal{T}_{i}} w\right)\right)-\left(\left[\bar{v}_{i}\right], n_{\mathcal{E}} \cdot\left\{w-P_{\mathcal{T}_{i}} w\right\}\right)_{\mathcal{E}}\right)=\lim _{i \rightarrow \infty}\left(G \bar{v}_{i}, P_{\mathcal{T}_{i}} w\right) \mathrm{d} t \\
= & \lim _{i \rightarrow \infty} \int_{0}^{T}\left(\left(G \bar{v}_{i}, w\right)+\left(G \bar{v}_{i}, P_{\mathcal{T}_{i}} w-w\right)\right) \mathrm{d} t=\lim _{i \rightarrow \infty} \int_{0}^{T}\left(G \bar{v}_{i}, w\right) \mathrm{d} t .
\end{aligned}
$$

The weak convergence in $L^{2}(\Omega)^{d}$ follows from density of smooth functions, cf. [Y80, p. 121].

A corresponding result holds for the approximate gradient in a stationary setting.

We now prepare the application of the Aubin-Lions lemma to dG approximation spaces. We construct a space $S$ with norm $\|\cdot\|_{S}$ which has the following properties: 
(S1) $\mathcal{S}^{p}(\mathcal{T})$ embeds continuously into $S$ with $\|v\|_{\mathcal{T}} \gtrsim\|v\|_{S}$ for all $v \in \mathcal{S}^{p}(\mathcal{T})$.

(S2) $S$ embeds compactly into $L^{2}(\Omega)$.

(S3) $S$ is a reflexive space.

It is well-known that the $\mathrm{dG}$ approximation spaces belong to $\mathrm{BV}(\Omega)$, e.g. [BO07]. For completeness we repeat that, without affecting the use of hanging nodes, one finds

$$
\|[v]\|_{L^{1}\left(\mathcal{E}_{\Omega}(\mathcal{T})\right)} \leq\left(\sum_{K \subset \mathcal{T}}\left\|h_{\mathcal{T}}\right\|_{L^{1}(\partial K)}\right)^{1 / 2}\left(\int_{\mathcal{E}_{\Omega}(\mathcal{T})} h_{\mathcal{E}}^{-1}[v]^{2} \mathrm{~d} x\right)^{1 / 2} \lesssim\left(\int_{\mathcal{E}_{\Omega}(\mathcal{T})} h_{\mathcal{E}}^{-1}[v]^{2} \mathrm{~d} x\right)^{1 / 2} .
$$

Now $\mathcal{S}^{p}(\mathcal{T}, \mathcal{P}) \subset \operatorname{BV}(\Omega)$ follows from

$$
\|v\|_{\mathrm{BV}(\Omega)} \leq\|v\|_{L^{1}(\Omega)}+\left\|\nabla_{h} v\right\|_{L^{1}(\Omega)}+\|[v]\|_{L^{1}\left(\mathcal{E}_{\Omega}(\mathcal{T})\right)} \lesssim\|v\|_{\mathcal{T}}, \quad v \in \mathcal{S}^{p}(\mathcal{T}, \mathcal{P}) .
$$

Because BV $(\Omega)$ embeds compactly into $L^{1}(\Omega)$ we may hope that (S2) can be established via (33). However, $\mathrm{BV}(\Omega)$ itself is not reflexive. In contrast, $L^{4}(\Omega)$, which is available via (M4), has the reflexivity property. Only, $L^{4}(\Omega)$ does not provide a suitable compactness argument. These observations motivate to search for a space which lies in an appropriate sense between $\mathrm{BV}(\Omega)$ and $L^{4}(\Omega)$.

Our starting point is that $L^{2}(\Omega)$ is gained by interpolating $L^{1}(\Omega)$ and $L^{4}(\Omega)$ with the complex method of Calderón and Lions. Two Banach spaces $X_{0}, X_{1}$ form a Banach couple $\left(X_{0}, X_{1}\right)$ if they are linearly and continuously embedded into a Hausdorff topological vector space. The space defined by complex interpolation with exponent $\theta \in(0,1)$ is denoted by $\left[X_{0}, X_{1}\right]_{\theta}$. We remark that if $X_{1}$ is reflexive then also $\left[X_{0}, X_{1}\right]_{\theta}$ is a reflexive space for $\theta \in(0,1)$, cf. [BL00, p. 449]. We shall make use of Theorem 11 from [CK95]:

Theorem 7. Consider the Banach couples $\left(X_{0}, X_{1}\right)$ and $\left(Y_{0}, Y_{1}\right)$ and a linear mapping $L: X_{0}+X_{1} \rightarrow Y_{0}+Y_{1}$ such that $\left.L\right|_{X_{0}}: X_{0} \rightarrow Y_{0}$ is compact and $\left.L\right|_{X_{1}}: X_{1} \rightarrow Y_{1}$ is continuous. Suppose there is a Banach space $Z$ such that $\left(Z, Y_{1}\right)$ is a Banach couple and such that $Y_{0}=\left[Z, Y_{1}\right]_{\alpha}$ for some $\alpha \in(0,1)$. Then $L:\left[X_{0}, X_{1}\right]_{\theta} \rightarrow$ $\left[Y_{0}, Y_{1}\right]_{\theta}$ is compact.

We choose $X_{1}=Y_{1}=L^{4}(\Omega)$. If there were a space $Z$ such that $L^{1}(\Omega)=\left[Z, Y_{1}\right]_{\alpha}$ then $L^{1}(\Omega)$ would be reflexive. Hence we make the alternative selection $Z=L^{1}(\Omega)$ and $Y_{0}=L^{4 / 3}(\Omega)$, taking into account that $L^{2}(\Omega)=\left[L^{4 / 3}(\Omega), L^{4}(\Omega)\right]_{1 / 2}$ and that $L^{4 / 3}(\Omega)=\left[L^{1}(\Omega), L^{4}(\Omega)\right]_{1 / 3}$. Every bounded sequence $\left(v_{i}\right)_{i}$ in the space $\operatorname{BV}(\Omega) \cap L^{4}(\Omega)$, which is equipped with the norm

$$
v \mapsto\|v\|_{\mathrm{BV}(\Omega)}+\|v\|_{L^{4}(\Omega)},
$$

has a subsequence $\left(\bar{v}_{i}\right)_{i}$ which strongly converges in $L^{1}(\Omega)$ to a function $v$ as $\mathrm{BV}(\Omega) \hookrightarrow L^{1}(\Omega)$ is compact. The boundedness of $\left(\bar{v}_{i}\right)_{i}$ in $L^{4}(\Omega)$ entails the weak convergence of a subsequence $\left(\overline{\bar{v}}_{i}\right)_{i}$ in $L^{4}(\Omega)$, whose limit also has to be $v$. Therefore $v \in L^{4}(\Omega)$. Application of the interpolation inequality

$$
\|w\|_{L^{4 / 3}(\Omega)} \leq\|w\|_{L^{1}(\Omega)}^{2 / 3}\|w\|_{L^{4}(\Omega)}^{1 / 3} \lesssim\|w\|_{\operatorname{BV}(\Omega)}^{2 / 3}\|w\|_{L^{4}(\Omega)}^{1 / 3}
$$

to $w=v-\overline{\bar{v}}_{i}$ proves that $\overline{\bar{v}}_{i} \rightarrow v$ strongly in $L^{4 / 3}(\Omega)$ as $i \rightarrow \infty$. Therefore $\mathrm{BV}(\Omega) \cap L^{4}(\Omega)$ is embedded compactly in $L^{4 / 3}(\Omega)$. The reflexivity of $L^{4}(\Omega)$ implies that

$$
S:=\left[\mathrm{BV}(\Omega) \cap L^{4}(\Omega), L^{4}(\Omega)\right]_{1 / 2}
$$

is reflexive. Theorem 7 ensures that $S=\left[X_{0}, X_{1}\right]_{1 / 2}$ with $X_{0}=\mathrm{BV}(\Omega) \cap L^{4}(\Omega)$ embeds compactly into $L^{2}(\Omega)$ and therefore $S$ satisfies (S1), (S2) and (S3). 


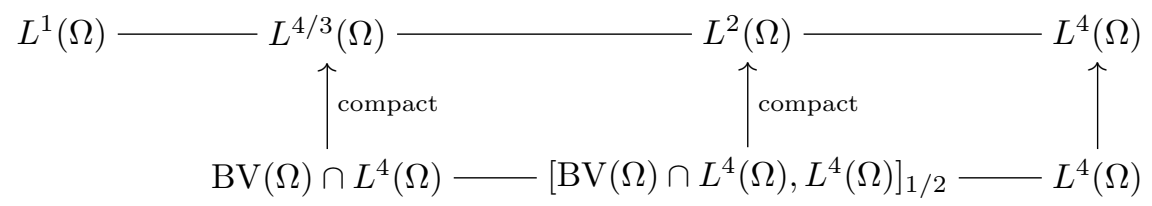

One benefit of $S$ is that we may apply the subsequent lemma with $B_{0}=S, B_{1}=L^{2}(\Omega)$ and $B_{2}=W^{p+1,4}(\Omega)$ without projecting discontinuous Galerkin solutions onto a conforming space, avoiding constraints which would otherwise lead to more restrictive approximation spaces and meshes.

Theorem 8 (Aubin-Lions, in [Z2]). Consider Banach spaces $B_{0}, B_{1}, B_{2}$ such that $B_{0} \hookrightarrow B_{1}$ is compact and $B_{1} \hookrightarrow B_{2}$ is continuous. Assume that $B_{0}$ and $B_{2}$ are reflexive. Then $W:=\left\{u \in L^{2}\left(0, T ; B_{0}\right): \partial_{t} u \in\right.$ $\left.L^{2}\left(0, T ; B_{2}\right)\right\}$ is compactly embedded into $L^{2}\left(0, T ; B_{1}\right)$.

Alternative to the construction of $S$ one can also apply the Aubin-Lions lemma in a conforming setting by considering the recovered approximations $R c_{h}$, cf. (7). However, changing meshes introduce a term $d_{t} R$ in the proof corresponding to Theorem 3. Also Galerkin orthogonality cannot be used as in (26), leading to less flexible meshes.

We note that for the construction of a space satisfying (S1) to (S3) a bound $\|v\|_{L^{2+\varepsilon}(\Omega)} \lesssim\|v\|_{\mathcal{T}_{c}^{j}}$, $\varepsilon>0$, is sufficient. The stronger condition (M4) is needed for Theorem 3.

\section{Convergence Analysis}

The question arises which choice of function spaces for the concentration $c$ should underly the convergence proof. Various approaches have been considered in the literature. For example, in a slightly different context, the spaces

$$
\left\{v \in L^{2}(0, T ; W(u)): \partial_{t} v \in L^{2}\left(0, T ; W(u)^{*}\right)\right\}, \quad W(u)=\left\{v \in H^{1}(\Omega):\left\|\mathbb{D}(u)^{1 / 2} \nabla v\right\|<\infty\right\}
$$

in [CE99]. We take a different route here, partially due to the issue whether smooth functions are dense in (35), e.g. [PC94], partially to remain within the framework of Bochner spaces. In light of the previous section and [F94], we select instead

$$
W:=\left\{w \in L^{2}(0, T ; S): \partial_{t} w \in L^{2}\left(0, T ; W^{p+1,4}(\Omega)^{*}\right)\right\} .
$$

Theorem 9. Let $\left(u_{i}, p_{i}, c_{i}\right)_{i \in \mathbb{N}}$ be a sequence of numerical solutions with $(\widetilde{h}, \widetilde{k}) \rightarrow 0$ as $i \rightarrow \infty$. Then there exists

$$
c \in L^{2}\left(0, T ; H^{1}(\Omega)\right) \cap H^{1}\left(0, T ; W^{p+1,4}(\Omega)^{*}\right)
$$

such that, possibly after passing to a subsequence,

$$
c_{i} \rightarrow c \quad \text { in } L^{2}\left(\Omega_{T}\right), \quad \partial_{t} \widetilde{c}_{i} \rightarrow \partial_{t} c \quad \text { in } L^{2}\left(0, T ; W^{p+1,4}(\Omega)^{*}\right), \quad \nabla c_{i} \rightarrow \nabla c \quad \text { in } L^{2}\left(0, T ; H^{-1}(\Omega)\right) .
$$

If $c_{i}^{0} \rightarrow c_{0}$ in $W^{p+1,4}(\Omega)^{*}$ at the initial time $t=0$ then $c$ satisfies (W3).

Proof. The weak convergence of $\partial_{t} \widetilde{c}_{i}$ in $L^{2}\left(0, T ; W^{p+1,4}(\Omega)^{*}\right)$ follows from the uniform bound on $\partial_{t} \widetilde{c}_{i}$ in Theorem 3. According to (S1) the injections $\mathcal{S}_{c}^{j} \hookrightarrow S$ are uniformly bounded with respect to the discretisation parameters. By (S2), (S3) and the Aubin-Lions lemma, the space $W$ embeds compactly into $L^{2}\left(\Omega_{T}\right)$. According to Theorems 2 and $3,\left(c_{i}\right)_{i}$ is bounded in $W$, ensuring the existence of a strongly converging subsequence. We shall from now on denote this subsequence by $\left(c_{i}\right)_{i}$. Theorems 4 and 2 guarantee $c \in L^{2}\left(0, T ; H^{1}(\Omega)\right)$ and the weak convergence of the gradient, noting that $\nabla c_{i}$ is bounded in $L^{2}\left(0, T ; H^{-1}(\Omega)\right)$ by (19) and (29). According 
to [DL5, p. 483] functions in $H^{1}\left(0, T ; W^{p+1,4}(\Omega)^{*}\right)$ belong to $\mathscr{C}\left(0, T, W^{p+1,4}(\Omega)^{*}\right)$. Due to the continuity of the trace operator in $\mathscr{C}\left(0, T, W^{p+1,4}(\Omega)^{*}\right)$ the function $c$ has a trace in $W^{p+1,4}(\Omega)^{*}$ which is equal to the $L^{\infty}(\Omega)$-function $c_{0}$.

The convergence of the Darcy velocity and the pressure is now a consequence of Strang's lemma.

Theorem 10. Let $\left(u_{i}, p_{i}, c_{i}\right)_{i \in \mathbb{N}}$ be a sequence of numerical solutions with $(\widetilde{h}, \widetilde{k}) \rightarrow 0$ and $c_{i} \rightarrow c$ in $L^{2}\left(\Omega_{T}\right)$ as $i \rightarrow \infty$. Then there exists a unique pair of functions $u \in L^{\infty}\left(0, T ; H_{N}(\operatorname{div} ; \Omega)\right)$ and $p \in L^{\infty}\left(0, T ; L_{0}^{2}(\Omega)\right)$ such that, possibly after passing to a subsequence,

$$
u_{i} \rightarrow u \quad \text { in } L^{\infty}\left(0, T ; H_{N}(\operatorname{div} ; \Omega)\right), \quad p_{i} \rightarrow p \quad \text { in } L^{\infty}\left(0, T ; L_{0}^{2}(\Omega)\right)
$$

as $(\widetilde{h}, \widetilde{k}) \rightarrow 0$. Furthermore, $(u, p, c)$ satisfies (W1).

Proof. Given $c \in L^{2}\left(\Omega_{T}\right)$ there is a unique pair $(u, p) \in L^{\infty}\left(0, T ; H_{N}(\operatorname{div} ; \Omega)\right) \times L^{\infty}\left(0, T ; L_{0}^{2}(\Omega)\right)$ solving, for all $v \in L^{\infty}\left(0, T ; H_{N}(\operatorname{div} ; \Omega)\right)$ and $q \in L^{\infty}\left(0, T ; L_{0}^{2}(\Omega)\right)$,

$$
\begin{aligned}
\int_{0}^{T}\left(\mu(c) \mathbb{K}^{-1} u, v\right) \mathrm{d} t-\int_{0}^{T}(p, \operatorname{div} v) \mathrm{d} t & =\int_{0}^{T}(\rho(c) g, v) \mathrm{d} t \\
\int_{0}^{T}(q, \operatorname{div} u) \mathrm{d} t & =\int_{0}^{T}\left(q^{I}-q^{P}, q\right) \mathrm{d} t,
\end{aligned}
$$

because this system satisfies the same inf-sup condition as (W1). Understanding (15) as a perturbation of (36), Strang's lemma for mixed finite element methods [BF91, p. 67] gives

$$
\begin{aligned}
&\left\|u-u_{i}\right\|_{L^{\infty}(0, T ; H(\operatorname{div} ; \Omega))}+\left\|p-p_{i}\right\|_{L^{\infty}\left(0, T ; L^{2}(\Omega)\right)} \lesssim \inf _{v_{h} \in \mathcal{S}_{u}}\left\|u-v_{h}\right\|_{L^{\infty}(0, T ; H(\operatorname{div} ; \Omega))} \\
& \quad+\inf _{q_{h} \in \mathcal{S}_{p}}\left\|p-q_{h}\right\|_{L^{\infty}\left(0, T ; L^{2}(\Omega)\right)}+\sup _{v_{h} \in \mathcal{S}_{u}} \frac{\left|\left(\mu(c) \mathbb{K}^{-1} u, v_{h}\right)_{\Omega_{T}}-\left(\mu\left(c_{i}\right) \mathbb{K}^{-1} u, v_{h}\right)_{\Omega_{T}}\right|}{\left\|v_{h}\right\|_{L^{\infty}(0, T ; H(\operatorname{div} ; \Omega))}} .
\end{aligned}
$$

We remark that the terms $M_{2 h}, M_{3 h}$ and $M_{4 h}$ in [BF91] vanish in our setting and that the reformulation (2.75) in [BF91] applies. Since pointwise $c_{i} \rightarrow c$ as $i \rightarrow \infty$ and since $\mu$ is bounded, the dominated convergence theorem implies that $\left\|\left(\mu(c)-\mu\left(c_{i}\right)\right) \mathbb{K}^{-1} u\right\| \rightarrow 0$. Hence the right-hand side of $(37)$ vanishes as $(\widetilde{h}, \widetilde{k}) \rightarrow 0$ and consequently $\left(u_{i}\right)_{i}$ and $\left(p_{i}\right)_{i}$ converge strongly.

We now show that the numerical solutions satisfy the concentration equation in the limit.

Theorem 11. Let $\left(u_{i}, p_{i}, c_{i}\right)_{i \in \mathbb{N}}$ be a sequence of numerical solutions with $(\widetilde{h}, \widetilde{k}) \rightarrow 0$ as $i \rightarrow \infty$ and let

$$
u \in L^{\infty}\left(0, T ; H_{N}(\operatorname{div} ; \Omega)\right), \quad p \in L^{\infty}\left(0, T ; L_{0}^{2}(\Omega)\right), \quad c \in L^{2}\left(0, T ; H^{1}(\Omega)\right) \cap H^{1}\left(0, T ; W^{p+1,4}(\Omega)^{*}\right)
$$

be a limit of $\left(u_{i}, p_{i}, c_{i}\right)_{i}$ in the sense of Theorems 9 and 10. Then $(u, c)$ satisfies (W2).

Proof. Step I: Let $v \in \mathscr{D}\left(0, T ; \mathscr{C}^{\infty}\left(\Omega_{T}\right)\right)$. Set $v_{i}(t):=P_{j} v(t)$ for $t \in\left(t_{j-1}, t_{j}\right]$, using the time steps and meshes associated to $c_{i}$. The strong convergence of $\left(\mathbb{D}_{h}\left(u_{i}\right)\right)_{i}$ in $L^{2}\left(\Omega_{T}\right)^{d \times d}$ follows from the Nemyckii trick [Z2, p. 39] and

$$
\lim _{i \rightarrow \infty}\left(\mathbb{D}(u)-\mathbb{D}_{h}\left(u_{h}\right)\right)=\lim _{i \rightarrow \infty}\left(\left(\operatorname{Id}-\Pi_{\mathcal{T}_{i}}\right)+\Pi_{\mathcal{T}_{i}}\right)\left(\mathbb{D}(u)-\Pi_{\mathcal{T}} \circ \mathbb{D}\left(u_{h}\right)\right)=0 .
$$

Using the strong convergence of $\left(\nabla_{h} v_{i}\right)_{i}$ in $L^{\infty}\left(\Omega_{T}\right)^{d}$ and the weak convergence of $\left(G c_{i}\right)_{i}$ in $L^{2}\left(\Omega_{T}\right)^{d}$, we find

$$
\begin{aligned}
\int_{0}^{T}(\nabla c, \mathbb{D}(u) \nabla v) \mathrm{d} t & =\lim _{i \rightarrow \infty} \int_{0}^{T}\left(\nabla c, \mathbb{D}_{h}\left(u_{i}\right) \nabla_{h} v_{i}\right) \mathrm{d} t=\lim _{i \rightarrow \infty} \int_{0}^{T}\left(G c_{i}, \mathbb{D}_{h}\left(u_{i}\right) \nabla_{h} v_{i}\right) \mathrm{d} t \\
& =\lim _{i \rightarrow \infty} \int_{0}^{T}\left(\nabla_{h} c_{i}, \mathbb{D}_{h}\left(u_{i}\right) \nabla_{h} v_{i}\right)-\left(n_{\mathcal{E}}\left[c_{i}\right],\left\{\mathbb{D}_{h}\left(u_{i}\right) \nabla_{h} v_{i}\right\}\right)_{\mathcal{E}_{\Omega}} \mathrm{d} t .
\end{aligned}
$$

From (10) and (24) it follows that

$$
\int_{0}^{T}\left(n_{\mathcal{E}}\left[v_{i}\right],\left\{\mathbb{D}_{h}\left(u_{i}\right) \nabla c_{i}\right\}\right)_{\mathcal{E}_{\Omega}} \mathrm{d} t \leq \int_{0}^{T}\left\|\mathbb{D}_{h}\left(u_{i}\right)^{1 / 2} \nabla c_{i}\right\|\left(1+\left\|u_{i}\right\|^{1 / 2}\right) \widetilde{h}^{1 / 2}\|v\|_{H^{2}(\Omega)}^{2} \mathrm{~d} t \rightarrow 0
$$


as $i \rightarrow \infty$. Similarly $(24)$ implies that $\int_{0}^{T}\left(\sigma^{2}\left[c_{i}\right],\left[v_{i}\right]\right)_{\mathcal{E}_{\Omega}} \mathrm{d} t$ vanishes as $i \rightarrow \infty$. Hence $B_{d}\left(c_{i}, v_{i} ; u_{i}\right)$ coincides in the limit with $(\nabla c, \mathbb{D}(u) \nabla v)$.

Step II: Let $v$ and $v_{i}$ be as in Step I. Recalling the definition of $q_{h}^{I}$ and $q_{h}^{P}$ from page 8

$$
\begin{aligned}
\int_{0}^{T}(u \cdot \nabla c, v) & +\left(q^{I} c, v\right) \mathrm{d} t=\int_{0}^{T}-(c, \operatorname{div}(u v))+\left(q^{I} c, v\right) \mathrm{d} t=\lim _{i \rightarrow \infty} \int_{0}^{T}-\left(c, \operatorname{div}\left(u_{i} v\right)\right)+\left(q^{I} c, v\right) \mathrm{d} t \\
& =\lim _{i \rightarrow \infty} \int_{0}^{T}-\left(c_{i}, \operatorname{div}\left(u_{i} v\right)\right)+\left(q^{I} c_{i}, v\right)+\sum_{K}\left(\left(c_{i}\right)^{+},\left(u_{i} \cdot n_{K}\right)_{+}[v]\right)_{\partial K} \mathrm{~d} t \\
& =\lim _{i \rightarrow \infty} \int_{0}^{T}\left(u_{i} \cdot \nabla_{h} c_{i}, v\right)+{ }^{1} / 2\left(\left(q^{I}+q_{h}^{I}+q^{P}-q_{h}^{P}\right) c_{i}, v\right)-\sum_{K}\left(\left(u_{i} \cdot n_{K}\right)_{-}\left[c_{i}\right], v^{+}\right)_{\partial K} \mathrm{~d} t \\
& =\lim _{i \rightarrow \infty} \int_{0}^{T} B_{c q}\left(c_{i}, v_{i} ; u_{i}\right)+B_{c q}\left(c_{i}, v-v_{i} ; u_{i}\right) \mathrm{d} t .
\end{aligned}
$$

The bound (20) ensures that $\left|B_{c q}\left(c_{i}, v-v_{i} ; u_{i}\right)\right| \lesssim \widetilde{h}^{1 / 2}|v|_{H^{2}(\Omega)}$, giving $\lim _{i} \int_{0}^{T} B_{c q}\left(c_{i}, v-v_{i} ; u_{i}\right) \mathrm{d} t=0$ and

$$
\int_{0}^{T}(u \cdot \nabla c, v)+\left(q^{I} c, v\right) \mathrm{d} t=\lim _{i \rightarrow \infty} \int_{0}^{T} B_{c q}\left(c_{i}, v_{i} ; u_{i}\right) \mathrm{d} t
$$

Step III: From

$$
\int_{0}^{T}\left(\phi c, \partial_{t} v\right) \mathrm{d} t=\lim _{i \rightarrow \infty} \int_{0}^{T}\left(\phi \widetilde{c}_{i}, \partial_{t} v\right) \mathrm{d} t=-\lim _{i \rightarrow \infty} \int_{0}^{T}\left(\phi \partial_{t} \widetilde{c}_{i}, v\right) \mathrm{d} t=-\lim _{i \rightarrow \infty} \int_{0}^{T}\left(\phi d_{t} c_{i}, v_{i}\right) \mathrm{d} t
$$

we conclude that

$$
\begin{gathered}
\int_{0}^{T}-\left(\phi c, \partial_{t} v\right)+(\mathbb{D}(u) \nabla c, \nabla v)+(u \cdot \nabla c, v)+\left(q^{I} c, v\right)-\left(\hat{c} q^{I}, v\right) \mathrm{d} t \\
=\lim _{i \rightarrow \infty} \int_{0}^{T}\left(\phi d_{t} c_{i}, v_{i}\right)+B_{d}\left(c_{i}, v_{i} ; u_{i}\right)+B_{c q}\left(c_{i}, v_{i} ; u_{i}\right)-\left(\hat{c} q^{I}, v_{i}\right) \mathrm{d} t=0 .
\end{gathered}
$$

Hence (W2) is satisfied for $v \in \mathscr{D}\left(0, T ; \mathscr{C}^{\infty}(\Omega)\right)$. The extension to $v \in \mathscr{D}\left(0, T ; W^{1,4}(\Omega)\right)$ follows from boundedness and density of smooth functions.

\section{Numerical Experiments}

The numerical experiments are carried out in two space dimensions on a mesh which consists of shape regular triangles without hanging nodes and which is not changed over time, that is $\mathcal{T}_{c}^{j}=\mathcal{T}_{u}^{j}=\mathcal{T}$. We use the lowest-order method, i.e. we have $p=1$ in (M2) and $\ell=0$ in (M3).

We adopt the commonly used diffusion-dispersion tensor [F94, CE99]

$$
\mathbb{D}(x, u)=\phi(x)\left(d_{m} \mathrm{Id}+|u| d_{\ell} E(u)+|u| d_{t}(\mathrm{Id}-E(u))\right),
$$

where $d_{m}$ is the molecular diffusion coefficient and $d_{\ell}$ and $d_{t}$ are the longitudinal and the transversal dispersion coefficients, respectively. The matrix $E(u)=u^{\top} u /|u|^{2}$ is the projection in $u$ direction. The concentration dependent viscosity is described by $\mu(c)=\mu(0)\left(1+\left(\mathcal{M}^{1 / 4}-1\right) c\right)^{-4}$, where $\mu(0)$ is the viscosity of oil and $\mathcal{M}=$ $\mu(0) / \mu(1)$ is the mobility ratio; the rescaled values for the parameters used below are taken from [WLELQ00]. The nonlinear system of equations arising in each time step is solved with a fixed-point iteration.

Numerical Example 1 (Qualitative Behaviour). The 'Quarter of Five Spot' benchmark [WLELQ00] models a regular pattern of injection and production wells in a horizontal reservoir of small thickness. On the computational domain $\Omega=(0,1)^{2}$ the injection well is located at $(1,1)$ and the production well is at $(0,0)$. The wells 

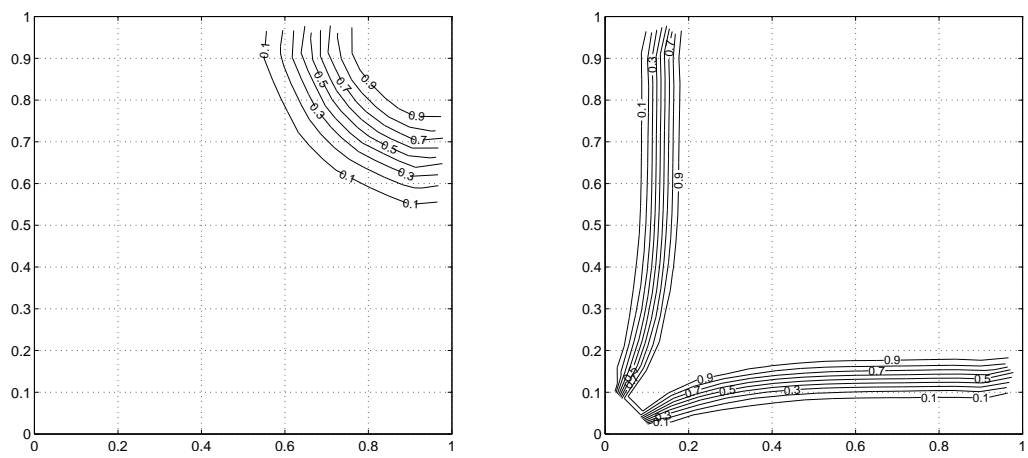

Figure 1: Contours of $c_{h}$ at time $t=2$ and $t=8$ for the linear, decoupled problem.

are represented by piecewise constant functions such that $\int_{\Omega} q^{I} \mathrm{~d} x=\int_{\Omega} q^{P} \mathrm{~d} x=0.018$. Furthermore, $\hat{c} \equiv 1$, $\mathbb{K} \equiv 0.0288 \mathrm{Id}, \phi \equiv 0.1, \mu(0)=1, d_{m}=3.6 \times 10^{-5}$ and $g \equiv 0$.

If the mobility ratio satisfies $\mathcal{M}=1$ and $d_{\ell}=d_{t}=0$ then the problem reduces to a decoupled, linear system. Figure 1 shows the contours of $c$ at $t=2$ and $t=8$. Initially the contours of $c$ evolve in nearly concentric circles, which is consistent with the nearly radial Darcy velocity field $u$ in the vicinity of the injection well and the isotropic character of $\mathbb{D}$. At later stages the effect of the no-flow boundary conditions and the production well becomes more pronounced, leading to a faster fluid flow along the domain diagonal.
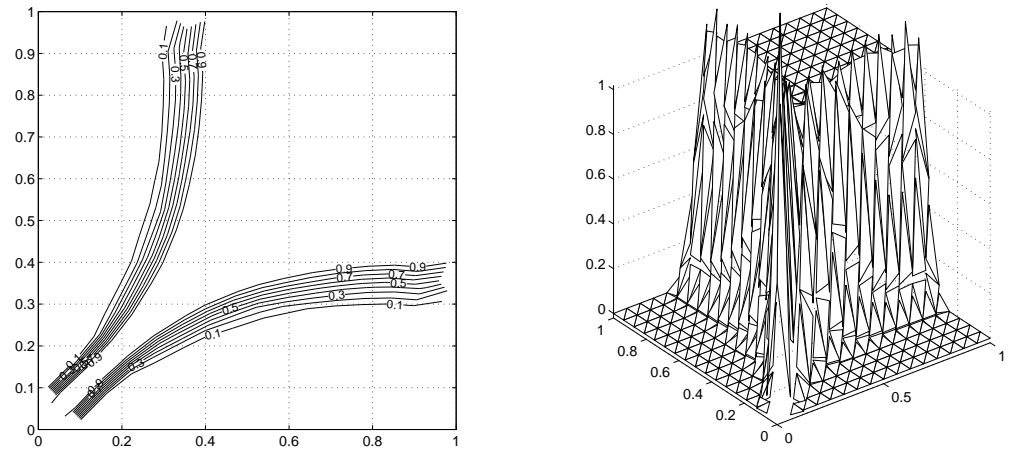

Figure 2: The concentration at time $t=5$ with anisotropic $\mathbb{D}$. Due to fingering, large areas of the reservoir are swept at a later time by the injected fluid.

If $\mathbb{D}$ is anisotropic, i.e. $d_{\ell} \neq d_{t}$, then $c$ typically exhibits viscous fingering on the macroscopic scale. For the computation depicted in Figure 2 we set $d_{m}=1.8 \times 10^{-6}, d_{\ell}=1.8 \times 10^{-4}$ and $d_{t}=1.8 \times 10^{-5}$. We use for the initial value $c_{h}^{0}$ a radial front around the injection well which is resolved by the mesh. Due to the large mobility ratio $\mathcal{M}=41$ the viscosity $\mu(c)$ changes rapidly across the concentration interfaces, leading there to an increased change in the Darcy velocity $u$. Taking also the large difference between longitudinal and transversal dispersion into account the fluid velocity is significantly increased along the domain diagonal compared to the decoupled linear case.

Numerical Example 2 (Singular Velocities). In the second numerical example we examine the effect of a singular velocity field, caused by a discontinuous permeability distribution and a re-entrant corner. The computational domain is L-shaped. As in the previous example, the injection well is located at $(1,1)$ and the production well is at $(0,0)$. The permeability is set to $\mathbb{K} \equiv 1.0 \times 10^{-6} \mathrm{Id}$ in the upper left quarter $(0,1 / 2) \times(1 / 2,1)$ of $\Omega$ and $\mathbb{K} \equiv 0.1 \mathrm{Id}$ elsewhere. Thus the porous medium is almost impenetrable in the 
upper left quarter, forcing a high fluid velocity at the reentrant corner where the nearly impenetrable barrier is thinnest.
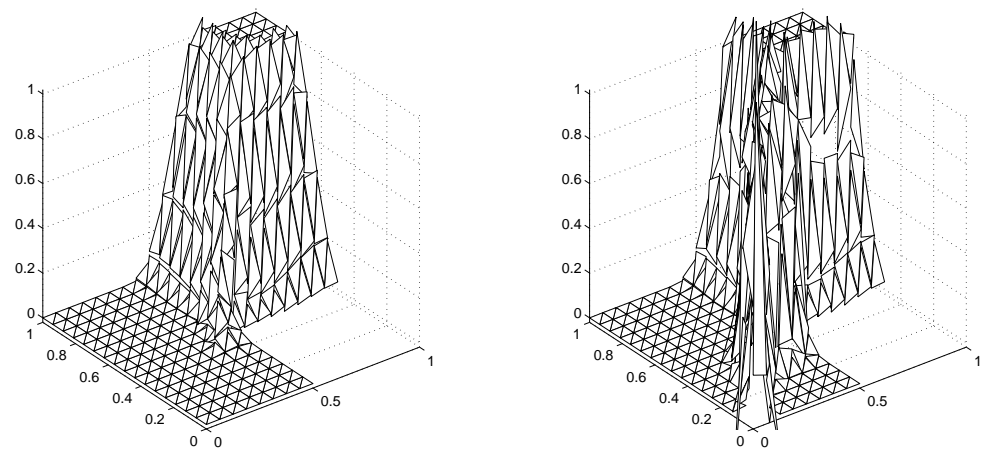

Figure 3: The concentration at $t=1.5$ and $t=2.5$.
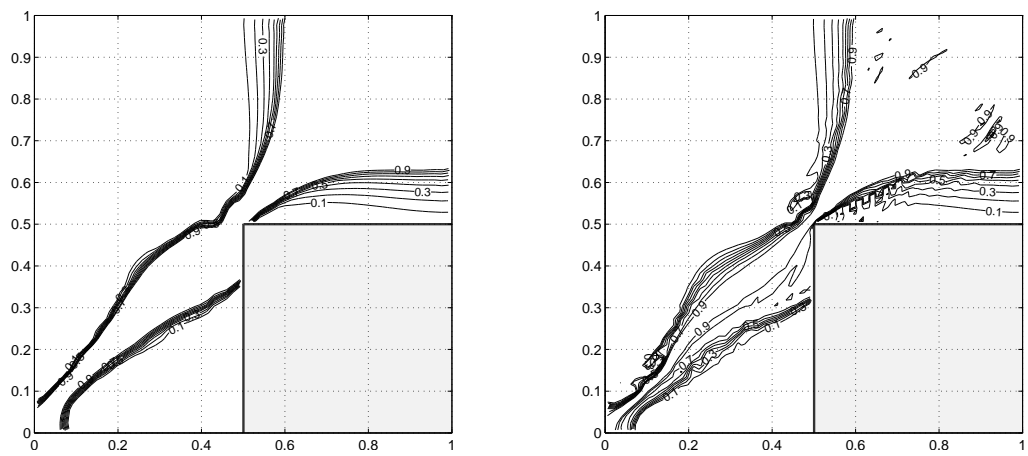

Figure 4: The concentration at time $t=3$ (left: $d G$ solution; right: conforming solution).

The experiment demonstrates that flux limiting is not needed. Figure 3 shows the concentration before and after the front passes the corner. The solution $c_{h}$ contains steep fronts but remarkably shows no more oscillations than in the first experiment. For comparison, we also solved the problem with conforming P1Lagrangian elements for $c$ combined with the Raviart-Thomas mixed method for $u$ and $p$. In this case, the oscillatory behaviour of the conforming approximation $c_{\mathrm{cg}}$ is substantially larger than that of $c_{h}$, cf. Figure 4 .

While the paper is concerned with the convergence of the method, our numerical experiments verify in accordance with Theorems 2 and 3 that the proposed finite element method remains stable in the presence of non-resolved singularities without the need for 'cut-off' functionals or super-penalisation.

Acknowledgement: We would like to thank M. Cwikel for his helpful comments on interpolation spaces. We acknowledge the support by the Deutsche Forschungsgemeinschaft (DFG) through the DFG Research Centre MATHEON 'Mathematics for key technologies' in Berlin as well as the support by the German Academic Exchange Service (DAAD) and the Indian Department of Science and Technology (DST) through the programme DAAD-DST PPP-05.

\section{References}

[ABCM02] D. Arnold, F. Brezzi, B. Cockburn, L.D. Marini, Unified analysis of discontinuous Galerkin methods for elliptic problems, SIAM J. Numer. Anal. 39(5):1749-1779, 2002. 
[AP98] M.A. Mohamed Ali, A. Pani, Mixed finite element methods for compressible miscible displacement problems in reservoir studies, Functional analysis with current applications in science, technology and industry (Aligarh, 1996), Pitman Res. Notes Math. Ser. 377:332-352, 1998.

[BL00] Y. Benyamini, J. Lindenstrauss, Geometric nonlinear functional analysis, AMS Colloquium Publications 48, 2000.

[BG88] C. Bernardi, V. Girault, A local regularization operator for triangular and quadrilateral finite elements, SIAM J. Numer. Anal. 35(5):1893-1916, 1988.

[BS02] S. Brenner, R. Scott, The mathematical theory of finite element methods, Texts in Applied Mathematics, vol. 15, Springer 2002.

[BF91] F. Brezzi, M. Fortin, Mixed and hybrid finite element methods, Springer Series in Computational Mathematics, vol. 15, Springer 1991.

[BO07] A. Buffa, C. Ortner, Variational convergence of IP-DGFEM, Technical Report 07/10, Oxford University Computing Laboratory, 2007.

[CE99] Z. Chen, R. Ewing, Mathematical analysis of reservoir models, SIAM J. Math. Anal. 30(2):431-453, 1999.

[CK95] M. Cwikel, N.J. Kalton, Interpolation of compact operators by the methods of Calderón and Gustavsson-Peetre, Proc. Edinburgh Math. Soc. (2), 38(2):261-276, 1995.

[DL5] R. Dautray, J-L. Lions, Mathematical analysis and numerical methods for science and technology, vol 5. Springer, 2000.

[DEW83] J. Douglas Jr., R.E. Ewing, M.F. Wheeler, A time-discretization procedure for a mixed finite element approximation of miscible displacement in porous media, RAIRO Anal. Numér. 17(3):249-265, 1983.

[EW80] R. Ewing, M. Wheeler, Galerkin methods for miscible displacement problems in porous media, SIAM J. Numer. Anal., 17(3):351-365, 1980.

[F94] X. Feng, On existence and uniqueness results for a coupled system modeling miscible displacement in porous media, J. Math. Anal. Appl. 194(3):883-910, 1995.

[F02] X. Feng, Recent developments on modeling and analysis of flow of miscible fluids in porous media, Fluid flow and transport in porous media: mathematical and numerical treatment (South Hadley, MA, 2001), Contemp. Math. 295:229-24, 2002.

[HSS02] P. Houston, C. Schwab, E. Süli, Discontinuous hp-finite element methods for advection-diffusion-reaction problems, SIAM J. Numer. Anal. 39(6):2133-2163, 2002.

[J05] M. Jensen, Discontinuous Galerkin methods for Friedrichs systems with irregular solutions, DPhil. thesis, Oxford, 2005.

[KP03] O. Karakashian, F. Pascal, A posteriori error estimates for a discontinuous Galerkin approximation of secondorder problems, SIAM J. Numer. Anal. 41(6):2374-2399, 2003.

[PC94] V.C. Piat, F.S. Cassano, Some remarks about the density of smooth functions in weighted Sobolev spaces, J. Convex Anal. 1(2):135-142, 1995, http://citeseer.ist.psu.edu/183113.html.

[RW02] B. Rivière, M. Wheeler, Coupling locally conservative methods for single phase flow, Comput. Geosci. 6(34):269-284, 2002.

[SRW02] S. Sun, B. Rivière, M. Wheeler, A combined mixed finite element and discontinuous Galerkin method for miscible displacement problem in porous media, Recent Progress in Computational and Applied PDEs, Kluwer/Plenum, 323-348, 2002.

[WLELQ00] H. Wang, D. Liang, R. E. Ewing, S. L. Lyons and G. Qin, An approximation to miscible fluid flows in porous media with point sources and sinks by an Eulerian-Lagrangian localized adjoint method and mixed finite element methods. SIAM J. Sci. Comput. 22(2):561-581, 2000.

[Y80] K. Yosida, Functional analysis, 6th edition, Springer, 1980.

[Z1] E. Zeidler, Nonlinear functional analysis and its applications, vol. 1. Springer 1986.

[Z2] E. Zeidler, Nonlinear functional analysis and its applications, vol. 2. Springer 1990. 